\title{
Risks For The Long Run And The Real Exchange Rate*
}

\author{
Riccardo Colacito ${ }^{\dagger}$ \\ Mariano M. Croce ${ }^{\ddagger}$
}

\begin{abstract}
Brandt, Cochrane, and Santa-Clara (2004) pointed out that the implicit stochastic discount factors computed using prices, on the one hand, and consumption growth, on the other hand, have very different implications for their cross country correlation. They leave this as an unresolved puzzle. We explain it by combining Epstein and Zin (1989) preferences with a model of predictable returns and by positing a very correlated long run component. We also assume that the intertemporal elasticity of substitution is larger than one. This setup brings the stochastic discount factors computed using prices and quantities close together, by keeping the volatility of the depreciation rate in the order of $12 \%$ and the cross country correlation of consumption growth around $30 \%$.
\end{abstract}

JEL classification: G12; G15; F31.

First Draft: October 31, 2004. This draft: October 31, 2006.

\footnotetext{
*We thank Dave Backus, Ravi Bansal, Eric Ghysels, Lars Hansen, Sidney Ludvigson, Fabrizio Perri, Adriano Rampini, our discussant at the NBER asset pricing program Adrien Verdelhan and in particular Tom Sargent for useful discussions and encouragement. We also thank seminar participants at NYU, Washington University in St.Louis, the New York Fed, Columbia, Rochester, Fordham, the Federal Reserve Board, the St.Louis Fed, Universitat Pompeu Fabra, UNC, UCSD, the Bank of Canada, SUNY-Albany, UCLA, Duke, the SED Meeting in Budapest, the Econometric Society World Congress in London, the Bocconi-NYU workshop in Florence, the NYU macro reading group and the University of Chicago-NYU college bowl for having provided useful comments. All errors remain our own.

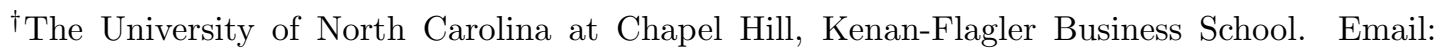
riccardo.colacito@unc.edu.

${ }_{\ddagger}^{\ddagger}$ New York University, Department of Economics. Email: massimiliano.croce@nyu.edu.
} 


\section{Introduction}

Ever since the seminal paper of Mehra and Prescott (1985), researchers have tried to reconcile the implications that prices and quantities have for asset returns. The heart of the problem is characterized by Hansen and Jagannathan (1991) that show that in a no arbitrage framework, stochastic discount factors should be at least as volatile as the highest Sharpe ratio of the return of any asset that they are intended to price. Financial data point in the direction of highly volatile stochastic discount factors, while in the context of an economy with a representative consumer with constant relative risk aversion (CRRA) preferences, quantities would imply lowly volatile stochastic discount factors, unless an unreasonably high coefficient of risk aversion is assumed. Brandt, Cochrane, and Santa-Clara (2004) extend the puzzle to a two country economy. They follow Backus, Foresi, and Telmer (1996) in showing that for any stochastic process for the depreciation rate and returns on domestic and foreign currency denominated assets, there exist stochastic discount factors, whose ratio is equal to the depreciation rate, provided that there are no arbitrage opportunities. This 'accounting' relationship allows them to retrieve the correlation of the stochastic discount factors from their volatilities and the standard deviation of the rate of growth of the exchange rate. Using the Hansen and Jagannathan (1991) bound the variance of the pricing kernels in major industrialized countries is in the order of $20 \%$ in annualized terms, while the volatility of the depreciation rate between the US and members of the same set of countries is typically between $11 \%$ and $15 \%$ also in annualized terms ${ }^{1}$. The implied correlation of the stochastic discount factors is not less than 0.96, when computed in this way. However data on consumption display a very low cross country correlation, that is generally not higher than 0.3 when the US is one of the two countries; the assumption that agents have CRRA preferences then imply a correlation of the stochastic discount factors of the same magnitude. The implication is that exchange rates will have to move around a lot to prevent arbitrage opportunities across countries: the volatility of the depreciation rate would have to take on values as high as $55 \%$, that is about 5 times what we observe in the actual data. This can be thought as an extension

\footnotetext{
${ }^{1}$ Source: IMF's 'International Financial Statistics'.
} 
of Mehra and Prescott (1985) equity premium puzzle: in a one country economy, consumption growth does not vary enough to keep track of the equity premium, while in a two country economy, consumption growth does not co-vary enough to explain the relative smoothness of the depreciation rate. We want to explain this puzzle.

This paper is at the crossroad of two growing bodies of literature. Starting with the contribution of Bansal and Yaron (2004), the class of models that includes long run risks has been successful in explaining some long standing puzzles of financial economics, including the afore mentioned high excess return of equities over the risk free rate $^{2}$. In the spirit of the line of research that applies the knowledge of closed economy consumption based asset pricing model to describing the dynamics of exchange rates ${ }^{3}$, this paper extends the framework proposed by Bansal and Yaron (2004) to a two country economy, each of them populated by a representative consumer with Epstein and Zin (1989) recursive preferences. We specify consumption growths as the sum of a slowly moving predictable component and an i.i.d. shock. We assume the former to be relatively smaller than the latter to respect the empirical finding that consumption growth is almost an i.i.d. process. We further assume that the predictable components are highly correlated across countries. This specification makes the puzzle disappear: we manage to obtain highly correlated stochastic discount factors along with a lowly volatile depreciation rate. This result is obtained in combination with consumption growth processes that are as volatile, as correlated and as persistent as observed in the actual data. We also show that the model can describe the international correlation of financial markets. The success of the model reflects the fact that when agents care about the timing of the resolution of uncertainty, long horizon consumption growth prospects are crucial determinants of stochastic discount factor processes. Having modeled these prospects as being highly correlated we obtain highly correlated stochastic discount factors. Because stochastic discount factors do most of the job of adjusting to avoid international arbitrage opportunities, exchange rates can take a lesser role and be less volatile.

What comes with the territory in these models is the difficulty of identifying

\footnotetext{
${ }^{2}$ Examples of these economies can be found in Bansal, Gallant, and Tauchen (2002), Hansen, Heaton, and Li (2004), Kiku (2006) and Croce, Lettau, and Ludvigson (2006).

${ }^{3}$ See for example, Lustig and Verdelhan (2006a) and Lustig and Verdelhan (2006b).
} 
the small predictable component of consumption growths in a formal econometric model. Bansal and Yaron (2004) show that the presence of a small predictable component in consumption growth cannot be rejected in the past century of US data and endowing the agents with sure knowledge of its presence helps explaining the behavior of asset returns. Bansal, Gallant, and Tauchen (2002) push the analysis one step forward by using price information to properly identify the properties of the consumption process. Our analysis follows in their footsteps as we demonstrate that consumption data alone contain insufficient evidence for the presence of low frequency components of consumption growths. However when the econometric system is enriched with restrictions imposed on the model by asset returns and exchange rates, we manage sharply to identify the departure of consumption growths from a purely i.i.d. process.

The paper is organized as follows. In section 2 we describe the determinants of the puzzle that we want to explain. In regards to the literature of which this is an extension, we denote it as the 'international equity premium puzzle'. In section 3, we write down a simple model that we can solve and calibrate. This provides a useful instrument to show the internal transmission mechanism of the economy. In section 4, we show how we can match a large number of statistics in the data. In section 5, we extend the model to include in each country a redundant asset that pays dividends, whose process looks like the aggregate dividend paid by the stock market and we explore what our model has to say about the international correlation of financial markets. The focus of section 6 is on the estimation of the model. We study the afore mentioned difficulties of providing conclusive evidence of predictable components of consumption growths from quantity data only and show the benefits of introducing prices in the picture. Section 7 concludes the paper, hinting to potential extensions of the model and summarizing the main findings.

\section{The 'international equity premium puzzle'}

We analyze two economies that we denote as home and foreign. Accordingly, we will index variables in the two countries with an $h$ and with an $f$. Assuming that the two countries are characterized by the absence of arbitrage opportunities, the 
following pricing condition has to be satisfied:

$$
E_{t}\left[\exp \left\{m_{t+1}^{f}\right\} R_{t+1}^{f}\right]=1=E_{t}\left[\exp \left\{m_{t+1}^{h}\right\} R_{t+1}^{h}\right]
$$

where $m_{t+1}^{h}$ and $m_{t+1}^{f}$ are the logarithms of the stochastic discount factors for returns denominated in home and foreign currencies respectively and $R_{t+1}^{h}$ and $R_{t+1}^{f}$ are gross returns. If the asset that in the foreign country delivers the return $R_{t+1}^{f}$ in expectation is also traded in the home country, then a feasible investment strategy consists in converting home prices into foreign prices at the spot exchange rate $e_{t}$ :

$$
E_{t}\left[\exp \left\{m_{t+1}^{f}\right\} R_{t+1}^{f}\right]=1=E_{t}\left[\exp \left\{m_{t+1}^{h}\right\} \frac{e_{t+1}}{e_{t}} R_{t+1}^{f}\right]
$$

Equation (1) gives us a connection between the rate of depreciation of the home currency and the two stochastic variables $m_{t+1}^{h}$ and $m_{t+1}^{f}$. Following Backus, Foresi, and Telmer (1996) and Brandt, Cochrane, and Santa-Clara (2004), we further assume that there is a complete set of markets for currencies and state contingent claims. This uniquely identifies the refinement of condition (1) as

$$
\pi_{t+1}=m_{t+1}^{f}-m_{t+1}^{h}
$$

where $\pi_{t+1}=\log \frac{e_{t+1}}{e_{t}}$. By taking the variance operator on both sides and by denoting $\sigma_{m^{i}}, \forall i \in\{h, f\}$ as the standard deviation of the stochastic discount factor in the two countries, $\rho_{m^{h}, m^{f}}$ as the correlation of the stochastic discount factors and $\sigma_{\pi}$ as the volatility of the depreciation rate, we obtain:

$$
\rho_{m^{h}, m^{f}}=\frac{\sigma_{m^{h}}^{2}+\sigma_{m^{f}}^{2}-\sigma_{\pi}^{2}}{2 \sigma_{m^{h}} \sigma_{m^{f}}}
$$

It is useful to restate the puzzle we are after in terms of equation (3). The Hansen and Jagannathan (1991) bound on the volatility of the logarithm of the stochastic discount factor ${ }^{4}$ is in the order of $39 \%$ in the US and $37 \%$ in the United Kingdom.

\footnotetext{
${ }^{4}$ Since the Hansen and Jagannathan (1991) bound is defined on the levels of the stochastic discount factors, we assume log-normality of the stochastic discount factors to derive the corresponding bound on $\sigma_{m^{h}}$ and $\sigma_{m^{f}}$.
} 
The standard deviation of the log-depreciation rate between the same countries is in the order of $11 \%$. These numbers and equation (3) imply a correlation of the stochastic discount factors of approximatively 0.96. In particular, it is shown in the appendix that the assumption of complete markets along with the Hansen and Jagannathan (1991) imply the following lower bound on the international correlation of stochastic discount factors.

Proposition 1. Let $\underline{\sigma}_{m^{h}}$ and $\underline{\sigma}_{m f}$ be the lower bounds on the volatilities of the stochastic discount factors in the home and in the foreign country, respectively. If markets are complete both domestically and internationally and the volatility of the depreciation of the home currency is strictly positive, then the following is the lower bound on the correlation of stochastic discount factors:

$$
\underline{\rho}_{m^{h}, m^{f}}=\left\{\begin{array}{lll}
\frac{\underline{\sigma}_{m}^{2}-\sigma_{\pi}^{2}}{\underline{\sigma}_{m} h \sqrt{\underline{\sigma}_{m^{h}}-\sigma_{\pi}^{2}}} & \text { if } & \underline{\sigma}_{m^{f}} \leq \underline{\sigma}_{m^{h}}-\sigma_{\pi}^{2} \\
\frac{\underline{\sigma}_{m h}^{2}+\underline{\sigma}_{m f}^{2}-\sigma_{\pi}^{2}}{2 \underline{\sigma}_{m} \underline{\sigma}_{m} f} & \text { if } & \underline{\sigma}_{m^{f}} \in\left(\underline{\sigma}_{m^{h}}-\sigma_{\pi}^{2}, \underline{\sigma}_{m^{h}}+\sigma_{\pi}^{2}\right) \\
\frac{\underline{\sigma}_{m f}^{2}-\sigma_{\pi}^{2}}{\underline{\sigma}_{m} \sqrt{\underline{\underline{g}}_{m}-\sigma_{\pi}^{2}}} & \text { if } & \underline{\sigma}_{m^{f}} \geq \underline{\sigma}_{m^{h}}-\sigma_{\pi}^{2}
\end{array}\right.
$$

Proof. See Appendix B.

This is what the data on prices have to say about the correlation of the stochastic discount factors in the US and in the UK. The puzzle arises by looking at the restrictions imposed by equation (3) when quantity data are used. In particular, assuming that the two countries have identical constant relative risk aversion (CRRA) preferences with coefficient of risk aversion $\gamma$, the log-stochastic discount factors are $m_{t}^{i}=-\gamma \Delta c_{t}^{i}, \forall i \in\{h, f\}$ and the correlation $\rho_{m^{h}, m^{f}}$ is simply the correlation of consumption growths. This number is about 0.3 that is far below the 0.96 calculated from financial data. Furthermore, consumption growth is notoriously poorly volatile, its standard deviation being $1.37 \%$ in the US and $2.83 \%$ in the UK. As a consequence, even if we were to set the coefficient of risk aversion to a number 


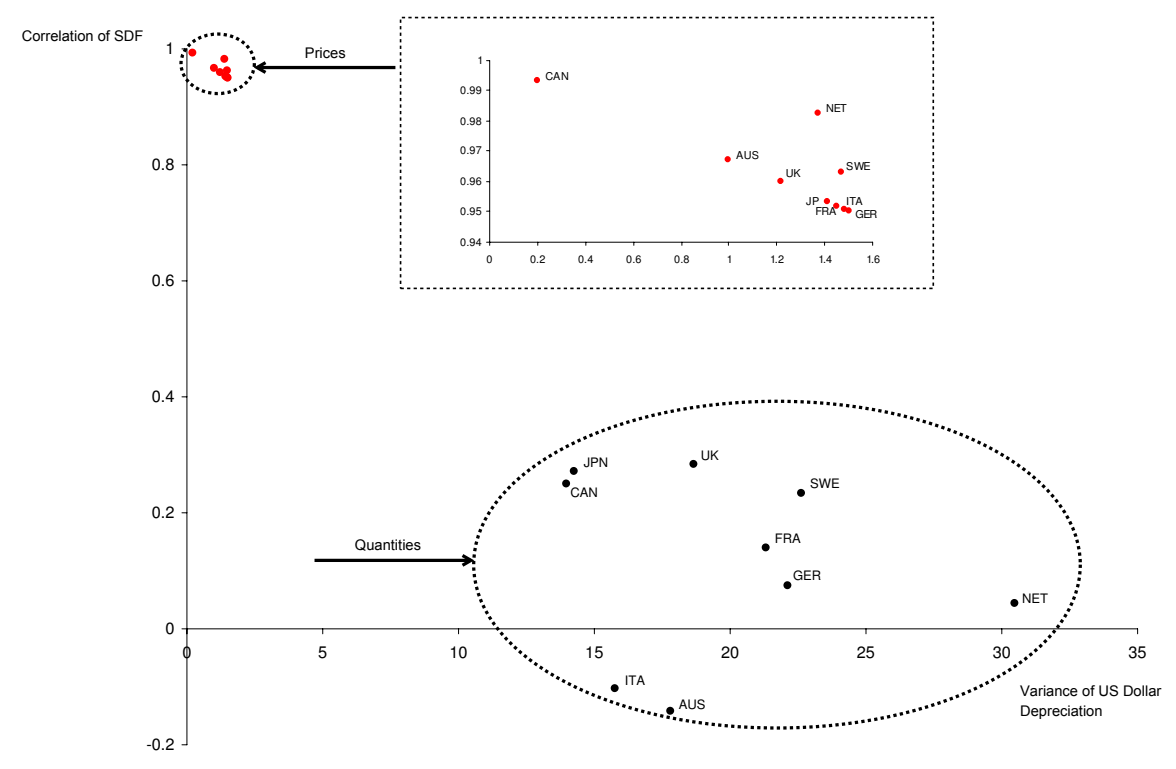

FIG. 1 - The puzzle in a cross section of countries. The horizontal axis reports the variance of the log depreciation rate and the vertical axis reports correlations. The home country is always the US. In the top left corner the variance-correlation of stochastic discount factors pairs are obtained from prices using the lower bound postulated in proposition 1 . In the bottom right part of the graph the variancecorrelation pairs are obtained from consumption data.

as high as 30 to reconcile lowly volatile consumption growths with highly volatile stochastic discount factors, equation (3) would point in the direction of an extremely high variance of the depreciation of the home currency: $18.6 \%$. Since this variance is about 15 times what we observe in the data it is natural to wonder whether there is something wrong with the observed exchange rate's fluctuations of US Dollars versus British Pounds or whether this particular version of consumption based asset pricing model is not suited to describe the data at hand ${ }^{5}$. We can also see this as a

\footnotetext{
${ }^{5}$ Brandt, Cochrane, and Santa-Clara (2004) examine market imperfections as the source of this discrepancy, showing that an unreasonable amount of extra volatility would need to be added to the stochastic discount factors to reconcile the two measure of correlations.
} 
restatement of Mehra and Prescott (1985) equity premium puzzle. In a one country model, consumption growth does not vary enough to explain the excess return over the risk free rate. In a two country model, consumption growth does not co-vary enough to keep track of movements in the exchange rate. This opens up to the rules of the game we want to play. We want to be able to reconcile the implications that both prices and quantities have for equation (3), by controlling at the same time for risk aversion, cross country correlation of consumption growth and volatility of the depreciation rate. In Figure 1 we show how the dichotomy prices-quantities extends also to other countries when paired with the US.

\section{Setup of the economy}

\subsection{Structure of the markets}

We study a two country endowment economy. We assume that there are only two goods in the whole economy and that these goods are country specific. To further simplify the setup, we impose that preferences are such that there is complete home bias, meaning that each country is willing to consume only the good that it is endowed with. Markets are complete, implying that returns are equalized across countries after accounting for the exchange rate. An equilibrium of this economy exists, in which each country behaves as in autarky both for consumption and asset holdings.

What is the meaning of the exchange rate in the absence of trade? If all assets are traded domestically and internationally, there must be Euler equations for holdings of any asset. The Euler equations for holdings of the same asset by a domestic and a foreign investor will then tie down the depreciation of the exchange rate in an arbitrage free environment. This is in the spirit of Lucas (1978) tree's model: although there is no trade going on in the economy, we can still give a price to any asset by reading it off the first order conditions. As an alternative, it is possible to think of the current setup of the model as the limiting case of an economy in which preferences are defined over a consumption aggregate that attaches a weight $\alpha$ to domestic goods and a weight $1-\alpha$ to foreign goods. The exchange rate is then defined 
as the relative price of the consumption aggregate in the two countries. We focus on the case in which $\alpha$ tends to zero. With standard time separable preferences it is trivial to show that this exchange rate exists (see for example Obstfeld and Rogoff (1996)). With the kind of preferences that we are using, the matter is more complicated, but following Anderson (2005) it can be shown to exist.

We motivate this extreme structure of the markets in at least three ways. First Brandt, Cochrane, and Santa-Clara (2004) assume this specification, too. As a matter of making our results comparable to theirs, it appears to be justified to follow in their footsteps. Second, it is a well documented fact that there is a marked home bias in consumption. Backus, Kehoe, and Kydland (1992) were the first to notice it and Lewis (1999) shows that there is almost a one for one response of consumption growth to domestic output growth after controlling for a world effect and heteroskedasticity. This appears to be true for G7 countries. Third, we want to use preferences of the Epstein and Zin (1989) type, that are recursive but not time separable and allowing for trades in the consumption goods market would severely complicate our analysis. There exists a literature that examines the dynamics of allocations when goods markets are open to trade and agents have non time separable preferences. Kan (1995) and Anderson (2005) provide analytical and theoretical tools to study these economies. In our case, the need to also introduce a slowly moving predictable component in the endowment process would make these frameworks difficult to extend. For the sake of explanation, we prefer to study the no-trade limiting case and leave a more realistic structure of the goods market for future extensions of this paper.

\subsection{Preferences and long run risks}

We model the two economies as each having a representative consumer with Epstein and Zin (1989) preferences:

$$
U_{t}^{i}=\left\{(1-\delta)\left(C_{t}^{i}\right)^{\frac{1-\gamma}{\theta}}+\delta\left[E_{t}\left[\left(U_{t+1}^{i}\right)^{1-\gamma}\right]\right]^{\frac{1}{\theta}}\right\}^{\frac{\theta}{1-\gamma}}, \forall i \in\{h, f\}
$$

where $\gamma$ is the coefficient of risk aversion and $\theta=\frac{1-\gamma}{1-1 / \psi}$ implicitly defines the intertemporal elasticity of substitution $\psi$. The two economies are assumed to be sym- 
metric, having the same preference and transition laws parameters. The implied pricing equation for the $j^{\text {th }}$ asset is

$$
E_{t}\left[M_{t+1}^{i} R_{j, t+1}^{i}\right]=1, \forall i \in\{h, f\}
$$

where the pricing kernel $M_{t+1}^{i}$ is a stochastic process that depends on consumption growth, $\frac{C_{t+1}^{i}}{C_{t}^{i}}$, on the return on the asset that pays consumption as its dividend, $R_{c, t+1}^{i}$ and on the preference parameters:

$$
\log M_{t+1}^{i}=\theta \log \delta-\frac{\theta}{\psi} \log \left(\frac{C_{t+1}^{i}}{C_{t}^{i}}\right)+(\theta-1) \log R_{c, t+1}^{i}, \forall i \in\{h, f\}
$$

In what follows, we will adopt the convention of denoting log-variables in small letters (hence $m_{t+1}^{i}=\log M_{t+1}^{i}$ ). We complete the system by specifying exogenous laws of motion for consumption growths as:

$$
\begin{aligned}
\Delta c_{t}^{i} & =x_{t-1}^{i}+\varepsilon_{c, t}^{i} \\
x_{t}^{i} & =\rho_{x} x_{t-1}^{i}+\varepsilon_{x, t}^{i}, \quad \forall i=\{h, f\}
\end{aligned}
$$

All shocks are i.i.d. normally distributed within each country, but they are allowed to be cross-country correlated according to the covariance matrix $\Sigma$ :

$$
\begin{aligned}
{\left[\begin{array}{llll}
\varepsilon_{c, t}^{h} & \varepsilon_{c, t}^{f} & \varepsilon_{x, t}^{h} & \varepsilon_{x, t}^{f}
\end{array}\right]^{\prime} } & \sim N(0, \Sigma) \\
\Sigma & =\sigma^{2}\left[\begin{array}{cc}
H_{c} & 0 \\
0 & \varphi_{e}^{2} H_{x}
\end{array}\right]
\end{aligned}
$$

where

$$
H_{c}=\left[\begin{array}{cc}
1 & \rho_{c}^{h f} \\
\rho_{c}^{h f} & 1
\end{array}\right] \quad H_{x}=\left[\begin{array}{cc}
1 & \rho_{x}^{h f} \\
\rho_{x}^{h f} & 1
\end{array}\right]
$$

In the appendix we show how to derive a first order linear approximation of the model. We obtain the following analytical expressions for stochastic discount 
factors, exchange rates and returns:

$$
\begin{array}{rlr}
m_{t+1}^{i} & =\log \delta-\frac{1}{\psi} x_{t}^{i}+\delta \frac{1-\gamma \psi}{\psi\left(1-\rho_{x} \delta\right)} \varepsilon_{x, t+1}^{i}-\gamma \varepsilon_{c, t+1}^{i} \\
\frac{e_{t+1}}{e_{t}} & =m_{t+1}^{f}-m_{t+1}^{h} \\
r_{c, t+1}^{i} & =\bar{r}_{c}+\frac{1}{\psi} x_{t}^{i}+\delta \frac{1-\frac{1}{\psi}}{1-\rho_{x} \delta} \varepsilon_{x, t+1}^{i}+\varepsilon_{c, t+1}^{i} & \\
r_{f, t+1}^{i} & =\bar{r}_{f}+\frac{1}{\psi} x_{t}^{i}, & \forall i \in\{h, f\}
\end{array}
$$

where $r_{f, t+1}^{i}$ is the log-risk free rate and $\bar{r}_{j}$ is the average return on asset $j, \forall j \in$ $\{c, f\}$. Given the system (8), the following two propositions can be stated.

Proposition 2. For a given choice of parameters and provided that $\rho_{x}^{h, f} \geq \rho_{c}^{h, f}$, the lowest cross country correlation of the stochastic discount factors is achieved for

$$
\psi=\frac{1}{\gamma} \widetilde{\delta} \quad, \quad \rho_{x}=0 \quad, \quad \rho_{x}^{h f}=\rho_{c}^{h f}
$$

where $\widetilde{\delta}=\frac{1-2 \rho_{x} \delta+\delta^{2}}{\delta^{2}\left(1-\rho_{x}^{2}\right)}$. Furthermore, if $\rho_{x}^{h, f}>\rho_{c}^{h, f}$, then $\left(\psi, \rho_{x}\right)=\left(\frac{1}{\gamma} \widetilde{\delta}, 0\right)$ is the unique minimizer.

Proof. See Appendix B.

Proposition 3. For a given choice of parameters, the lowest volatility of the depreciation rate is achieved for $\rho_{x}^{h f}=1$.

Proof. See Appendix B.

Propositions 2 and 3 are intended to stress that three crucial ingredients are needed in order to obtain highly correlated stochastic discount factors together with lowly volatile exchange rates. First of all we need to break the tight relationship between risk aversion and intertemporal elasticity of substitution, that constant relative risk aversion preferences would otherwise impose. Indeed, as the subjective discount factor $\delta$ approaches unity, as it is the case when the model is calibrated to a monthly or quarterly decision problem, the minimum correlation of stochastic discount factors is obtained for a value of intertemporal elasticity of substitution 
that is arbitrarily close to the reciprocal of the coefficient of risk aversion. This is independent of the calibration of the rest of the model. Hence it does not seem implausible to think of the contribution of Brandt, Cochrane, and Santa-Clara (2004) as positing a lower bound on what the correlation of stochastic discount factors in a consumption based asset pricing model would look like. Proposition 2 is silent about agents preferring early or late resolution of uncertainty ${ }^{6}$. In the next section we will show that any departure from constant relative risk aversion preferences will do the job of obtaining highly correlated stochastic discount factors. However we can anticipate that we will not want to make the intertemporal elasticity of substitution too small, as this would result in the counterfactual outcome of infinitely volatile returns, as suggested by the system of equations (8).

Proposition 3 stresses the role of the predictable components of consumption growths. The intuition is straightforward. Epstein and Zin (1989) preferences introduced an extra term in the formula for the stochastic discount factors in the two countries, in the form of the return that pays the consumption bundle at each period. The price of this asset reflects the total expected flow of dividends, in our case consumption, that it entitles to. Since we model consumption as having a predictable component, it is natural to conclude that this term will have an impact on the return of the asset that will be proportional to its persistence. By appropriately raising the correlation of the $x_{t}$ 's we will manage to increase the correlation of the returns on the consumption assets and ultimately the correlation of stochastic discount factors. This decreases the role of the exchange rate in eliminating international arbitrage opportunities. In the next section we show how it is only the combination of all the three ingredients put forward by propositions 2 and 3 that delivers our result.

\section{Results from a calibrated economy}

\subsection{Choice of parameters}

In this section we report the results of a calibrated economy of the type discussed earlier. We assume that the countries share the same calibration, as reported in

\footnotetext{
${ }^{6}$ Epstein and Zin (1989) argue that an agent prefers early resolution of uncertainty if $\psi>1 / \gamma$, while late resolution is preferred if $\psi<1 / \gamma$.
} 
Table 1. As the structure of our two parallel economies mimics those discussed by Bansal and Yaron (2004) and Bansal, Gallant, and Tauchen (2002) most of the coefficients used in our analysis are either estimated or calibrated in those papers. We choose our model to described a monthly decision problem and as a consequence we set the subjective discount factor to 0.998. In terms of proposition 2 , this means that the correlation of stochastic discount factors is minimized for values of $\psi \approx 1 / \gamma$. We set the coefficient of risk aversion $\gamma$ equal to 4.25 , that is a number relatively low compared to what is commonly found in the equity premium puzzle literature and with the number proposed by Brandt, Cochrane, and Santa-Clara (2004) in their extension to an international context. The intertemporal elasticity of substitution, $\psi$ is equal to 2 and is the one estimated by Bansal, Gallant, and Tauchen (2002). As far as the calibration of the parameters of the laws of motion of consumption growth is concerned, our goal is to reproduce the average behavior of consumption growth is the United States and in the United Kingdom. We set $\rho_{x}=.987$, that is the value estimated by Bansal, Gallant, and Tauchen (2002) and that is slightly higher than the 0.979 calibrated by Bansal and Yaron $(2004)^{7}$. The standard error of the idiosyncratic shock to the predictable components is extremely small compared to the one of the idiosyncratic shock to consumption growth, allowing the latter to be the main determinant of the volatility of consumption growth. This is in the spirit of a large part of the literature that models consumption growth as an almost i.i.d. process (see among others Tallarini (2000)). The standard deviation of consumption growth implied by our choice of parameters is approximatively $2.4 \%$ in annualized terms, that is in between the average growth of per capita consumption of nondurables and services from 1970 to 1998 for the US and the UK.

The choice of the correlations coefficients is driven by the need of matching key features of the data. We set $\rho_{x}^{h f}$ to 1 as suggested by Proposition 3 to keep the volatility of the depreciation rate to about $11-12 \%$. The cross country correlation of the idiosyncratic shocks to consumption is chosen so to obtain a correlation of consumption growth in the order of 0.3 . We will show that the results are robust to relaxing the assumption of symmetrically calibrated countries in the context of the

\footnotetext{
${ }^{7}$ We show in section 6 that this assumption cannot be rejected on the grounds of the available data for the US and the UK.
} 
TABLE 1

Baseline CAlibration.

\begin{tabular}{llc}
\hline \hline$\mu_{c}$ & Average consumtpion growth & $15 \times 10^{-4}$ \\
$\Psi$ & Intertemporal elasticity of substitution & 2 \\
$\gamma$ & Risk aversion & 4.25 \\
$\delta$ & Subjective discount factor & 0.998 \\
$\rho$ & Autoregressive coefficient of the long run component $x_{t}$ & 0.987 \\
$\phi_{e}$ & Ratio of long run shock and short run shock volatilities & 0.048 \\
$\sigma$ & Standard error of the short run shock to consumption (in \%) & $68 \times 10^{-4}$ \\
$\rho_{x}^{h f}$ & Cross country correlation of the long run shock & 1.0 \\
$\rho_{c}^{h f}$ & Cross country correlation of the short run shock to consumption & 0.3 \\
\hline
\end{tabular}

Notes - The two countries share the same calibration.

estimation exercise that we run in section 6 .

\subsection{Correlation of stochastic discount factors}

In the previous section we have discussed how it is the combination of long run risks, Epstein-Zin preferences and cross country highly correlated $x_{t}$ to drive the results. Figure 2 details this finding with regard to the correlation of stochastic discount factors. Using equation (8), we study how this correlation varies with the coefficient of intertemporal substitution. In each of the two panels, the dark line is drawn according to our baseline calibration. In light of Proposition 1, we are not surprised that the minimum of this graph happens in the vicinity of $\psi \approx 1 / \gamma$. By moving away from $1 / \gamma$ in either direction, there is a sharp increase in the correlation of stochastic discount factors. However, as stressed in the discussion of the two propositions, increasing the correlation of stochastic discount factors by lowering the intertemporal elasticity of substitution would have the unappealing side effect of pushing the volatilities of returns to infinity. It is also for this reason that in our baseline calibration, we set $\psi=2$, that leaves us with a correlation that is above 0.9 , as price data seem to suggest. Interestingly enough, we do not need to take a stand on the intertemporal elasticity of substitution being greater or smaller than 1 , as far as our goal of obtaining highly correlated stochastic discount factors is concerned. Figure 2 shows that any $\psi$ larger than 0.5 would do the job of making the stochastic 
discount factors of the two countries at least $80 \%$ correlated, provided that the remaining coefficients are calibrated as in Table 1 . This comment deserves particular attention, given that the intertemporal elasticity of substitution being greater or smaller than 1 has given rise to a long lasting debate in the literature. Ignoring the presence of stochastic volatility, Hall (1988) and Lustig and Van Nieuwerburgh (2005) estimate this number to be below unity. Guvenen (2005) points out that limited participation in the stock market is able to produce findings consistent with both capital and consumption fluctuations as long as most of the wealth is held by a small fraction of population with a high elasticity of intertemporal substitution. Attanasio and Weber (1989) use the Family Expenditure Survey to document an intertemporal elasticity of substitution in excess of one in the UK. This paper follows the tradition of the risks for the long run literature in calibrating $\psi=2$ with the objective of appropriately describing the first two moments of returns, as it will be discussed in the next two sections.

What happens if we perturb our baseline calibration? The left panel of Figure 2 shows that decreasing the correlation of the long run shocks would have the consequence of noticeably decreasing also the correlation of stochastic discount factors. This effect comes through a reduced correlation of the returns on the consumption asset, as we document in the last subsection of our calibration exercise. The right panel of Figure 2 shows that reducing the persistence of the predictable components of consumption growths in the two countries would also largely affect the success of our analysis. Once again, the reduced correlation of stochastic discount factors would come from a reduced correlation in returns. The intuition is that if we reduce the persistence of the predictable part of consumption growth, then the stream of dividends paid by the consumption asset is mainly affect by the idiosyncratic shock to consumption, whose international correlation is quite low. So why do not increase the persistence of the predictable components? Because, by doing this we would increase (at an increasing rate) the share of consumption growth variance that is explained by its predictable component, eventually contradicting the empirical evidence on consumption growth being almost an i.i.d. process. In the figures, we do not report the behavior of the variables of interest when changing the coefficient of risk aversion, but we refer to proposition 2 to explain what would happen: given 

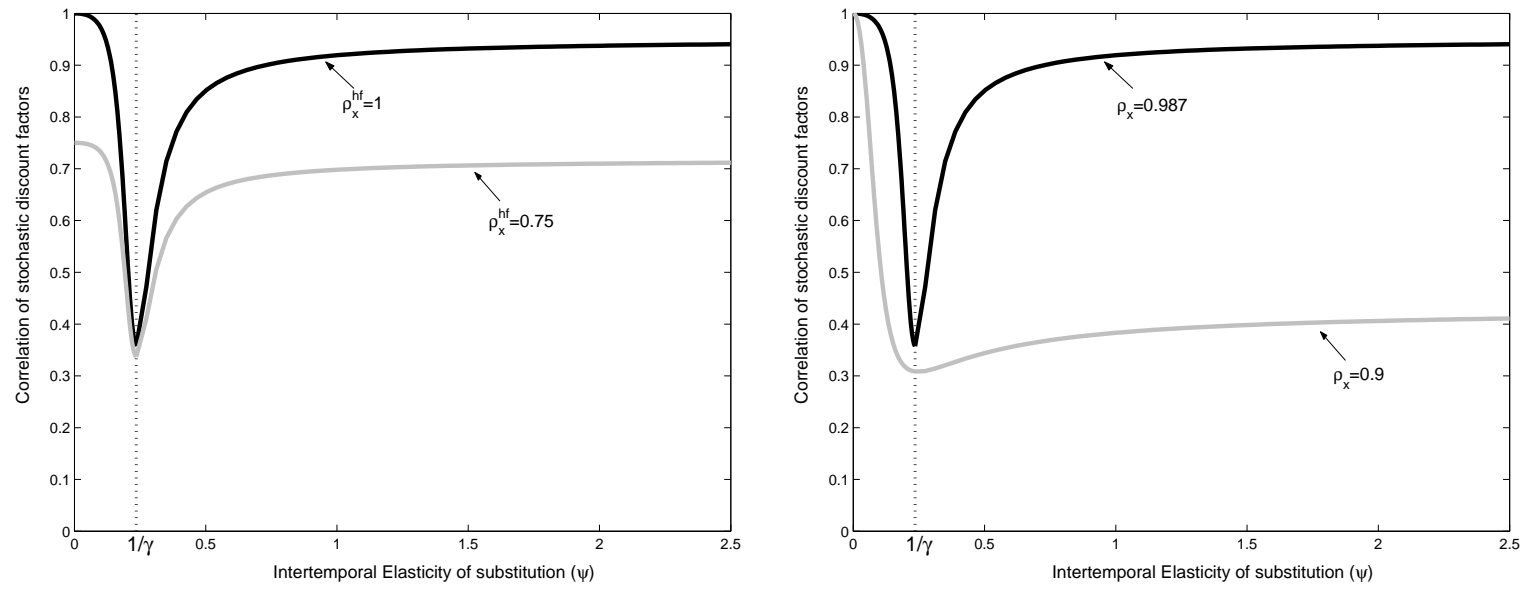

FIG. 2 - The role of intertemporal elasticity of substitution. In both panels, the dark line reports the correlation of stochastic discount factors when $\psi$ changes. The grey line on the left panel is drawn for a smaller value of $\rho_{x}^{h f}$ everything else equal and the grey line on the right panel is drawn for a lower $\rho_{x}$.

that the subjective discount factor is close to 1, lowering (increasing) the coefficient of risk aversion would move the minimum of the correlation of stochastic discount factors to the right (left) in Figure 2.

\subsection{Volatility of the depreciation rate}

As formulated in section 2, the puzzle has two parts: one is the correlation of stochastic discount factors that we discussed in the previous subsection and the other one is the volatility of the depreciation rate that we analyze here. Figure 3 plots the volatility of the depreciation rate against the coefficient of risk aversion. The two horizontal dashed lines represent the region in which the volatility of the depreciation rate typically falls for major industrialized countries. In our baseline calibration we set $\gamma=4.25$ that leaves us with a volatility that is well within the region of interest, as documented by the dark lines in the two panels of Figure 3. Increasing the risk aversion of the representative consumers of the two countries to the high levels impelled by the equity premium puzzle literature would push the volatility to counterfactual levels similar to those that motivated Brandt, Cochrane, and Santa-Clara (2004) to postulate the existence of a puzzle. Also in this case, 

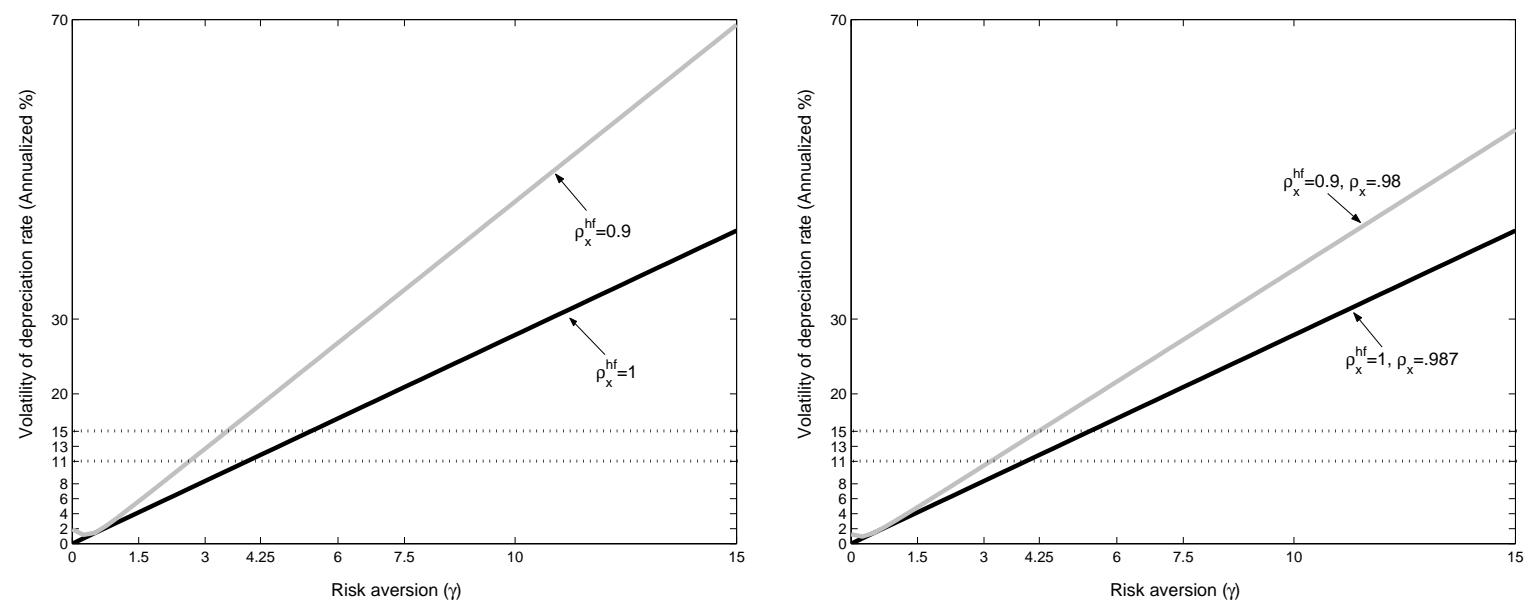

FIG. 3 - The role of risk aversion. In both panels, the dark line reports the volatility of the depreciation rate when $\gamma$ changes. The grey line in the left panel is drawn for a smaller value of $\rho_{x}^{h f}$ everything else equal and the grey line in the right panel is drawn for lower $\rho_{x}$ and $\rho_{x}^{h f}$.

our calibration proves itself crucial. Reducing the correlation of the long run shocks is going to reduce the correlation of stochastic discount factors as we documented before and this will open up to a greater role for the exchange rate in avoiding international arbitrage opportunities. The left panel of Figure 3 shows that unless agents are made less risk averse, we would be left with too much volatility of the depreciation rate at the calibrated level of risk aversion. This could be offset if were also to decrease the persistence of the predictable components, as it would result in the volatility of the stochastic discount factors to fall more than their international correlation. However, the evidence reported in figure 2 refrains us from taking this action and even more so considering the implications that a lowly volatile stochastic discount factor would have for asset returns.

\subsection{The role of returns}

Table 2 reports the behavior of other relevant variables as we increase the persistence of the long run component. As already observed, the correlation of the stochastic discount factors is increasing and even more so the closer we get to $\rho_{x}=1$. The standard deviation of consumption growth does not change too much, except for the 
TABLE 2

The Role Of Returns.

\begin{tabular}{ccccccccc}
\hline \hline$\rho_{x}$ & $\rho_{m^{h}, m^{f}}$ & $\sigma_{\pi}$ & $\sigma_{\Delta c}$ & $\sigma_{\varepsilon_{c}} / \sigma_{\Delta c}$ & $\sigma_{r_{c}}$ & $\rho_{r_{c}^{h}, r_{c}^{f}}$ & $\rho_{\Delta c^{h}, \Delta c^{f}}$ & $\rho_{\Delta c^{f}, r_{c}^{h}}$ \\
\hline 0.00 & 0.30 & 11.85 & 2.36 & 1.00 & 2.36 & 0.30 & 0.30 & 0.30 \\
0.70 & 0.31 & 11.85 & 2.36 & 1.00 & 2.36 & 0.31 & 0.30 & 0.30 \\
0.90 & 0.41 & 11.85 & 2.37 & 0.99 & 2.43 & 0.34 & 0.31 & 0.30 \\
0.987 & 0.94 & 11.85 & 2.46 & 0.92 & 4.88 & 0.84 & 0.35 & 0.16 \\
0.999 & 1.00 & 11.85 & 3.46 & 0.46 & 44.75 & 1.00 & 0.67 & 0.03 \\
\hline
\end{tabular}

Notes - All figures are annualized. All coefficients are set to the numbers reported in Table 1 , except for $\rho_{x}$ that takes the values reported in the first column.

case in which $\rho_{x}=0.999$. This is the result of our choice of setting the volatility of the long run term to a tiny number compared to the volatility of $\varepsilon_{c}$. Indeed the ratio $\sigma_{\varepsilon_{c}} / \sigma_{\Delta c}$ indicates that the contribution of long run risks to the volatility of consumption growth is always small unless $\rho_{x} \approx 1$. The two covariance terms that seem to be driving the results are the correlation of the consumption assets and the correlation of consumption growth and consumption asset returns across countries. As the persistence of the $x_{t}$ components rises, the returns on the consumption asset will mainly reflect the long run perspectives of consumption growth, implying a low correlation with $\Delta c_{t}$ in any country, that is driven for a large part by the short run shock $\varepsilon_{c}$, as we have already discussed before. By the same token, returns are going to be increasingly correlated across country as a result of our choice of setting $\rho_{x}^{h f}$ to one. Since these returns enter the stochastic discount factors of the two countries, a considerably higher correlation of the two can be achieved under our benchmark calibration. Last, it is interesting to notice how our choice of setting the correlation of the long run components to one, as suggested by proposition 3, manages to keep the volatility of the depreciation rate to a constant $11.85 \%$.

\section{Other moments of international financial mar- kets}

In this section, we run a validation exercise in which we ask whether the model can also account for other features of international financial markets. To this end, 
we retain all the assumptions that we made so far about agents preferences and about consumption growths containing small but highly persist and highly crosscountry correlated predictable components and we introduce two assets (one for each country), whose dividends mimic those of a value weighted portfolio in the US and in the UK. Specifically we assume that the growths of dividends follow a process that is similar to the one that we have used for consumption growth:

$$
\Delta d_{t}^{i}=\mu_{d}+\lambda x_{t-1}+\varepsilon_{d, t}^{i}, \forall i \in\{h, f\}
$$

with $\varepsilon_{d, t}^{i}$ i.i.d. normal with mean zero and variance $\left(\sigma \varphi_{d}\right)^{2}, \forall i \in\{h, f\}$. The coefficient $\lambda$ is referred to as the leverage and is usually set to a number larger than 1 . The six shocks of the economy follow a multivariate normal process with covariance matrix $\widetilde{\Sigma}$ :

$$
\begin{aligned}
{\left[\begin{array}{llllll}
\varepsilon_{c, t}^{h} & \varepsilon_{c, t}^{f} & \varepsilon_{x, t}^{h} & \varepsilon_{x, t}^{f} & \varepsilon_{d, t}^{h} & \varepsilon_{d, t}^{f}
\end{array}\right]^{\prime} } & \sim N(0, \widetilde{\Sigma}) \\
\widetilde{\Sigma} & =\left[\begin{array}{cc}
\Sigma & 0 \\
0 & \sigma^{2} \varphi_{d}^{2} H_{d}
\end{array}\right]
\end{aligned}
$$

where

$$
H_{d}=\left[\begin{array}{cc}
1 & \rho_{d}^{h f} \\
\rho_{d}^{h f} & 1
\end{array}\right]
$$

and $\Sigma$ is defined as in the previous section. To better describe the nonlinearities in returns we employ a numerical algorithm to solve for the price-dividend and price-consumption ratios. The Appendix describe in details the procedure.

The introduction of the dividends process requires the calibration of four additional parameters. Our guidelines in choosing these coefficients will be to appropriately describe the first two moments of dividend growth, the cross country correlation of dividend growth and the leverage in the US and the UK.

Table 3 reports the baseline calibration of $\lambda^{i}, \mu_{d}^{i}, \varphi_{d}^{i}$ and $\rho_{d}^{h f}, \forall i \in\{h, f\}$. By construction the variance of dividend growth explained by its predictable component is very small and in the order of $3 \%$. The coefficient $\lambda$ is set in such a way that the ratio $\sigma_{\Delta d} / \sigma_{\Delta c}$ is in the range $(4,8)$ estimated by Ludvigson, Lettau, and Wachter 
TABLE 3

Calibration Of Additional Parameters.

\begin{tabular}{llr}
\hline \hline$\lambda$ & Leverage & 3.0 \\
$\mu_{d}$ & Average dividend growth & .0007 \\
$\varphi_{d}$ & Volatility ratio of short run shocks to dividend and consumption growth & 5.0 \\
$\rho_{d}^{h f}$ & Cross country correlation of the short run shock to dividends & -0.1 \\
\hline
\end{tabular}

Notes - The coefficients refer to a monthly calibration. The remaining coefficients are calibrated according to Table 1.

(2004). In our baseline calibration we set $\lambda=3.0$ that implies $\sigma_{\Delta d} / \sigma_{\Delta c}=4.86$. The cross country correlation of the short run shocks to dividends, $\rho_{d}^{h f}$, is set to target the almost zero correlation of dividend growths between the US and the UK.

Table 4 shows that our baseline calibration is able to match key features of international financial markets. Stochastic discount factors that have the property of being highly correlated produce excess returns whose first two unconditional moments are in the range that we observe for the US and the UK. This framework also delivers high average and low volatility excess returns. Bansal and Yaron (2004) showed that this class of models had the potential of appropriately describing this moments in the US. We extend their analysis to show that a similar setup can also explain the same moments in the United Kingdom in the context of symmetric calibration exercise. We push our analysis one step forward, as we demonstrate that the model can also match the average correlation of excess returns in the two countries. This is accomplished in spite of the low calibrated correlation of dividend growths. Instead a model with time-separable CRRA preferences would deliver returns that are as cross-country correlated as the growth of the dividends that they entitle to are. We regard this result as further validation of the existence of highly cross country correlated predictable components of consumption growth. One dimension along which our model fails to replicate the data is the correlation of risk free rates. Even though we use a numerical solution of the model to construct the moments reported in Table 4, a linear approximation of the model can help explaining the result. The Appendix shows that the approximate solution for the risk free rates in this economy is a linear function of the predictable component of consumption growth only and 
TABLE 4

INTRODUCING DIVIDENDS.

\begin{tabular}{|c|c|c|c|c|}
\hline & & $\overline{\mathrm{US}}$ & UK & Model \\
\hline$\rho\left(m^{h}, m^{f}\right)$ & Correlation of SDF & - & - & 0.93 \\
\hline$\sigma\left(\frac{e_{t+1}}{e_{t}}\right)$ & Volatility of depreciation rate & \multicolumn{2}{|c|}{11.21} & 11.83 \\
\hline$E\left(r_{d}-r_{f}\right)$ & Average excess return & 7.02 & 9.17 & 7.01 \\
\hline$\sigma\left(r_{d}-r_{f}\right)$ & Volatility of excess return & 17.13 & 22.83 & 19.60 \\
\hline$\rho\left(r_{d}^{h}-r_{f}^{h}, r_{d}^{f}-r_{f}^{f}\right)$ & Correlation of excess returns & \multicolumn{2}{|c|}{0.60} & 0.58 \\
\hline$E\left(r_{f}\right)$ & Average risk free rate & 1.47 & 1.62 & 1.33 \\
\hline$\sigma\left(r_{f}\right)$ & Volatility of risk free rate & 1.53 & 2.92 & 1.19 \\
\hline$\rho\left(r_{f}^{h}, r_{f}^{f}\right)$ & Correlation of risk free rates & \multicolumn{2}{|c|}{0.65} & 1.00 \\
\hline$\sigma\left(r_{c}\right)$ & Volatility of return on cons. & - & - & 4.74 \\
\hline$\rho\left(r_{c}^{h}, r_{c}^{f}\right)$ & Correlation of returns on cons. & _ & - & 0.85 \\
\hline$\sigma(\Delta c)$ & Volatility of consumption growth & 1.37 & 2.86 & 2.45 \\
\hline$\frac{\sigma^{2}(x)}{\sigma^{2}(\Delta c)} \times 100$ & Share of predictable cons. variance & - & - & 8.18 \\
\hline$\sigma(\Delta d)$ & Volatility of dividend growth & 16.85 & 6.87 & 11.96 \\
\hline$\rho\left(\Delta c^{h}, \Delta c^{f}\right)$ & Correlation of consumption growth & \multicolumn{2}{|c|}{0.28} & 0.35 \\
\hline$\rho\left(\Delta d^{h}, \Delta d^{f}\right)$ & Correlation of dividend growth & \multicolumn{2}{|c|}{-0.03} & -0.07 \\
\hline
\end{tabular}

Notes - All figures are annualized. All coefficients are set to the numbers reported in Table 1 and Table 4.

as a consequence the correlation of risk free rates will entirely reflect the perfect correlation of the $x$ 's. If on the one hand this can be regarded as a failure of the model, on the other hand this invites us to reflect on the importance of risk free rates and other yields to the extent that the econometrician wants to identify the low frequency component of consumption growth. Two recent papers study the role of yields in economies with long run risks. Piazzesi and Schneider (2006) show that modeling inflation as also containing a low frequency component that is negatively correlated with the one of consumption growth can deliver an upward sloping nominal term structure of the interest rate when agents have a preference for the timing of the resolution of uncertainty. Colacito (2006) proposes a generalization of the framework analyzed in this paper that includes more than one predictable component of consumption growth in each country, that has the potential of reconciling the correlation of risk free rates that we observe in the data and that we obtain from 
the model.

\section{$6 \quad$ Estimating long run risks}

The previous sections have shown that it is the combination of three crucial ingredients that can address the puzzle raised by Brandt, Cochrane, and Santa-Clara (2004). In particular, we need to disentangle the intertemporal elasticity of substitution from the coefficient of risk aversion and we need to model consumption growth has having a highly persistent and cross country correlated predictable component. Can we justify the assumptions that we made about the consumption processes on the grounds of the data that we have? The literature (Bansal and Yaron (2004) and Hansen, Heaton, and Li (2004)) has typically struggled to find conclusive evidence of the consumption process that we adopt in our model, when the focus is on the US only. The two panels of Figure 4 report the estimated spectra and coherence ${ }^{8}$ of US and UK consumption growth (continuous lines) along with the theoretical periodograms and the $95 \%$ confidence intervals (dashed lines) under the alternative assumptions that consumption growth is i.i.d. (left panel) and that it has a small predictable component (right panel). The latter are computed simulating 1000 independent samples according to model (5) with the calibration of Table 1 expect for $\sigma_{c}^{h}, \sigma_{c}^{f}$ and $\rho_{c}^{h f}$, that are set so that the simulated series have the same sample variance and covariance of the actual series. The main finding of Figure 4 is that the two alternative assumptions look equally likely to say the least, as the estimated spectra and coherence typically lie within the $95 \%$ confidence intervals. Quantity data are not enough to identify the low frequency components of consumption growths ${ }^{9}$.

\footnotetext{
${ }^{8}$ See appendix Appendix D for details.

${ }^{9}$ In the working paper version, we show how to use the Kalman filter to obtain a recursive characterization of the likelihood function starting from the state space representation for consumption growths. The estimated parameters are typically characterized by very large confidence intervals and this finding remains true even when a large cross-section of countries is employed. These results are available upon request to the authors.
} 


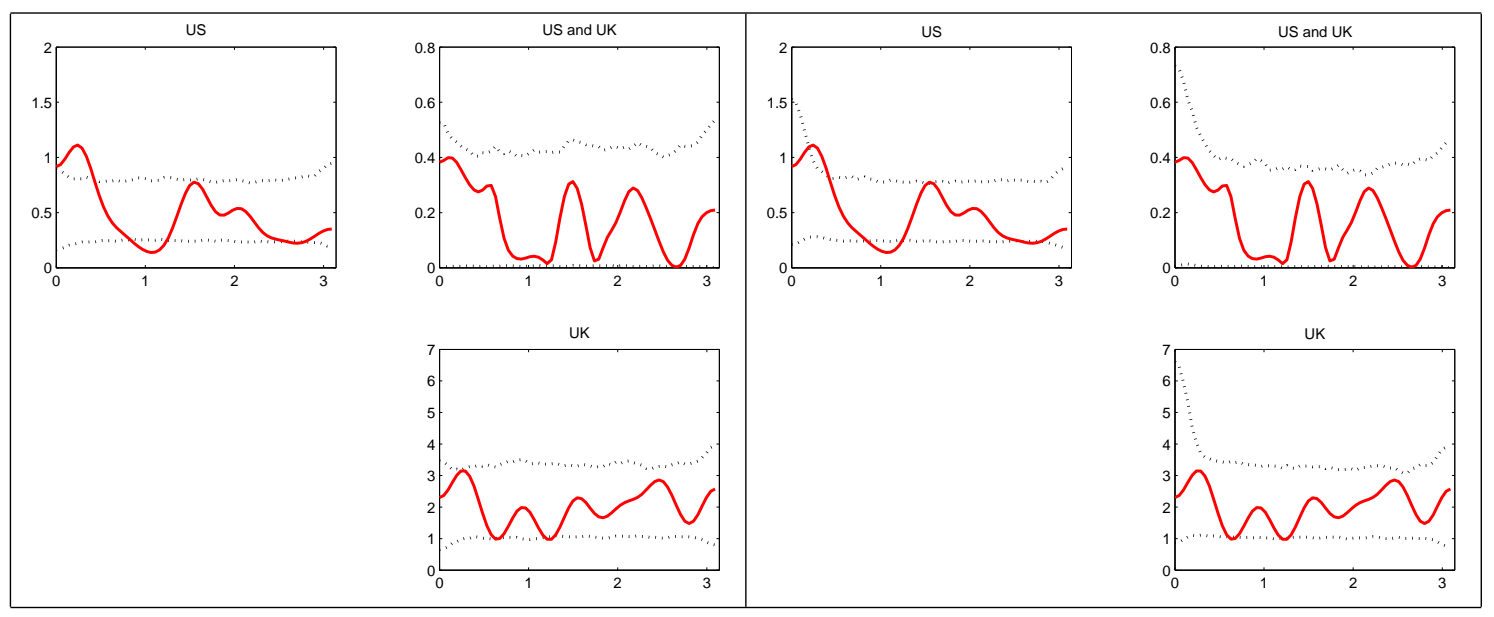

FIG. 4 - Sample periodogram. The grey line is the data sample periodogram, while the dashed lines represent the $95 \%$ confidence interval.

\subsection{The data}

We use the same data set of Campbell (2003). Data are quarterly observations on private consumption of nondurables and services obtained from NIPA for the US and from IMF's 'International Financial Statistics' (henceforth IFS). These quantities are deflated using the Consumer Price Index provided by CRSP for the United States and by IFS for the other countries. The common sample spans from 1970.1 to 1998.4. In the last part of this section we also use price data. In particular, the real US Dollar-British Pound Exchange Rate is derived as the ratio of the price index in UK currency and the MSCI Price Index in dollar terms. The risk free rates are T-bill rates from CRSP for US and the rate at which 91-day T-bills are allotted in the UK. Stock market returns and dividends are for value-weighted portfolios obtained from CRSP for the US and from Morgan Stanley Capital Perspective.

\subsection{A GMM based estimation}

The spirit of this paper and of the literature that it belongs to is to try to understand the properties of the consumption process that would allow us to price assets in a no arbitrage framework. Even though these characteristics of consumption cannot be clearly identified from quantity data alone, exchange rates and asset returns should help in this task. One possibility would be to use the linearized version of the model 
described in the appendix and obtain a recursive representation of the likelihood function by applying the Kalman filter. Although our model has been shown to provide a good description of several unconditional moments of international data, it is still unsatisfactory along a number of dimensions. The correlation of risk free rates and the one between consumption differentials and exchange rates would require a more general setup as the one studied by Colacito (2006). Furthermore it is extremely hard to account for the conditional distributions of exchange rates and excess returns even in the presence of stochastic volatility, as documented among others by Backus and Smith (1993) and Bansal and Yaron (2004). Also, our assumption of complete home bias in consumption and of complete markets are likely to provide an additional source of noise in exchange rates fluctuations. For all these reasons, we implement a GMM style estimation, in which we only focus on the set of moments that are the object of the attention of this paper. Alternatively this can be interpreted as a way of providing statistical support to our calibration described in Tables 1 and 3. We restrict our attention to the linearized version of the model as summarized by the system of equations (E.3)-(E.5) reported in the Appendix.

In particular, we focus on a total of 46 moments conditions to identify 16 coefficients, as detailed in the appendix. Due to the analytical difficulty of computing the quarterly counterparts of these moments starting from a model that is specified at a monthly frequency, we follow Hansen and Sargent (2006) in assuming that model and data are observed at the same frequency. We then check that the moments of interest agree quantitatively with the numbers that we reported in the earlier sections. One more caveat is brought into the problem by the fact that we need to constrain the correlation coefficients to be bounded by 1 in absolute value. Andrews (2002) warns that theoretically when the true parameter is on the boundary, GMM estimators are not asymptotically normally distributed ${ }^{10}$. The preliminary investigation that we conducted in the earlier sections suggested that the correlation of the predictable components of consumption growths may be equal to one. This happened to be the case also when we attempted to estimate this correlation along

\footnotetext{
${ }^{10}$ In particular: if only one parameter is on the boundary, that parameter is going to have a half-normal distribution, while the distribution of all other parameters will be non-standard and obtainable only via simulations.
} 
TABLE 5

PRICES AND LONG RUN RISKS.

\begin{tabular}{|c|c|c|c|c|}
\hline & \multicolumn{2}{|c|}{ Consumption only } & \multicolumn{2}{|c|}{ Whole Model } \\
\hline & Point Estimate & Std Err & Point Estimate & Std Err \\
\hline$\rho^{h}$ & 0.912 & $(0.121)$ & 0.996 & $(0.003)$ \\
\hline$\rho^{f}$ & 0.970 & $(0.053)$ & 0.961 & $(0.026)$ \\
\hline$\varphi_{e}^{h}$ & 0.131 & $(0.109)$ & 0.013 & $(0.007)$ \\
\hline$\varphi_{e}^{f}$ & 0.126 & $(0.112)$ & 0.118 & $(0.042)$ \\
\hline$\sigma^{h}$ & 0.546 & $(0.055)$ & 0.296 & $(0.066)$ \\
\hline$\sigma^{f}$ & 1.184 & $(0.112)$ & 0.551 & $(0.122)$ \\
\hline$\rho_{c}^{h f}$ & 0.219 & $(0.106)$ & 0.204 & $(0.343)$ \\
\hline$\psi^{h}$ & - & - & 1.842 & $(0.222)$ \\
\hline$\psi^{f}$ & - & - & 1.534 & $(0.276)$ \\
\hline$\gamma^{h}$ & - & - & 9.000 & $(3.776)$ \\
\hline$\gamma^{f}$ & - & - & 7.692 & $(3.535)$ \\
\hline$\lambda^{h}$ & - & - & 8.990 & $(4.055)$ \\
\hline$\lambda^{f}$ & - & - & 3.179 & $(0.493)$ \\
\hline$\varphi_{d}^{h}$ & - & - & 7.073 & $(0.263)$ \\
\hline$\varphi_{d}^{f}$ & - & - & 1.564 & $(0.110)$ \\
\hline$\rho_{d}^{h f}$ & - & - & -0.114 & $(0.057)$ \\
\hline & \multicolumn{4}{|c|}{ Unconditional moments (at point estimates) } \\
\hline$\sigma\left(\frac{e_{t+1}}{e_{t}}\right)$ & \multicolumn{2}{|c|}{-} & \multicolumn{2}{|c|}{10.787} \\
\hline$\rho\left(m^{h}, m^{f}\right)$ & \multicolumn{2}{|c|}{-} & \multicolumn{2}{|c|}{0.970} \\
\hline$\rho\left(r_{d}^{h}, r_{d}^{f}\right)$ & \multicolumn{2}{|c|}{-} & \multicolumn{2}{|c|}{0.420} \\
\hline
\end{tabular}

Notes - The second and third columns report the results of the estimation on consumption data only, while the fourth and fifth columns report the results of the estimation of the whole model. In parenthesis we report the standard errors. The bottom panel of the table reports the unconditional moments in annualized terms of the variables of interest using the point estimates. The subjective discount factors $\delta^{h}$ and $\delta^{f}$ are equal to the numbers reported in Table 1 raised to the third power to account for the quarterly frequency of the model. The following relabeling is used to simplify the estimation routine: $\sigma_{x}^{i}=\phi_{e} \sigma_{c}^{i}$ and $\sigma_{d}^{i}=\varphi_{d}^{i} \sigma_{c}^{i}$. All parameters that refer to standard errors are multiplied by 100 . 
with the other parameters of the mode ${ }^{11}$. To overcome the complications that this would bring in terms of asymptotic distribution of the estimators, we decided to set $\rho_{x}=1$ and to focus on the conditional distribution of the other parameters. Since none of these parameters happens to be on the boundary, the results of Hansen (1982) apply.

Table 5 shows the results of the estimation. As a matter of comparison, the left side of the table reports what we obtain when only the moments restrictions relative to consumption data are imposed. Given our preliminary investigations, it should not come as a surprise that when this is the case, it is hard to reject the hypothesis that consumption growths are pure i.i.d. processes, as documented by the large standard errors associated with the volatility parameters of the predictable components. Furthermore, the autoregressive coefficients $\rho^{h}$ and $\rho^{f}$ are also characterized by a significant degree of uncertainty, that would have important consequences in terms of the pricing implications discussed in the previous sections. The last two columns of the table show that adding price restrictions helps in tightening the confidence intervals of the point estimates of these set of parameters around the values that we specified in the theoretical analysis of the model. The two intertemporal elasticity of substitution are significantly larger than unity, as this is necessary in order to explain the high equity premium in the US and in the UK. The bottom part of the table shows that even though the parameters in the two countries are no longer set to the same values and the model is specified at a quarterly frequency, it is still possible to account for the moderate degree of volatility of the depreciation rate of the US dollar and for the degree of cross country correlation of both the stochastic discount factors and the stock market returns. This adds a degree of robustness to the theoretical results described in the previous sections.

\section{Concluding remarks}

We have shown that allowing for an intertemporal elasticity of substitution larger than one and for a persistent and highly cross country correlated forecastable component of consumption growth in the economy described by Brandt, Cochrane, and

\footnotetext{
${ }^{11}$ These results are available upon requests to the authors.
} 
Santa-Clara (2004) it is possible to reconcile the measure of international correlation of stochastic discount factors obtained from data on consumption and from data on prices. This result is achieved in combination with a lowly volatile depreciation rate and without requiring a high correlation of the consumption processes and high coefficients of risk aversion. We have also shown how key features of the data can be described by the same parametrization that allows us to meet our primary goal, extending in this way the set of properties of the models that take into account long run risks beyond what pointed out by Bansal and Yaron (2004).

Future developments of this line of research should address the deeper economic questions of where the predictable components of consumption growth come from in the context of a fully specified production economy, of what happens when the assumption of complete home bias is relaxed and of providing additional evidence of consumption growth predictability. We are optimistic about the positive findings of this paper to hold also in more general setups that have the potential of extending even further the set of properties of this class of models. 


\section{References}

Anderson, E. (2005). The dynamics of risk-sensitive allocations. Forthcoming in Journal of Economic Theory.

Andrews, D. W. (2002). Generalized method of moments estimation when a paramter is on a boundary. Journal of Business and Economic Statistics 20(4), 530-544.

Attanasio, O. P. and G. Weber (1989). Intertemporal substitution, risk aversion and the euler equation for consumption. The Economic Journal, Supplement: Conference Papers. 99, 59-73.

Backus, D., S. Foresi, and C. Telmer (1996, June). Affine models of currency pricing. NBER Working Papers 5623.

Backus, D. and G. Smith (1993). Consumption and real exchange rates in dynamic exchange economies with nontraded goods. Journal of International Economics 35, $297-316$.

Backus, D. K., P. J. Kehoe, and F. E. Kydland (1992). International real business cycles. Journal of Political Economy 100(4), 745-775.

Bansal, R., A. R. Gallant, and G. Tauchen (2002). Rational pessimism and exuberance. Working Paper, Department of Economics, University of North Carolina, Chapel Hill NC 27599-3305 USA.

Bansal, R. and A. Yaron (2004). Risks for the long run: A potential resolution of asset pricing puzzles. The Journal of Finance 59, 1481-1509.

Bartlett, M. (1964). The spectral analysis of two-dimensional point processes. Biometrika 51, 299-311.

Brandt, W., J. Cochrane, and P. Santa-Clara (2004). International risk sharing is better than you think (or exchange rates are much too smooth). Forthcoming Journal of Monetary Economics.

Campbell, J. (2003). Consumption-based asset pricing. forthcoming in Handbook of the Economics of Finance. George Constantinides, Milton Harris, and Rene Stulz eds., North-Holland, Amsterdam.

Campbell, J. Y. and R. J. Shiller (1988). Stock prices, earnings, and expected dividends. The Journal of Finance XLIII (3), 661-676.

Colacito, R. (2006). Six puzzles looking for a model. a unified framework for international finance puzzles. UNC, unpublished manuscript.

Croce, M. M., M. Lettau, and S. Ludvigson (2006). Investor information, long-run risk, and the duration of risky cash flows. NYU, unpublished manuscript.

Epstein, L. G. and S. E. Zin (1989, July). Substitution, risk aversion, and the temporal behavior of consumption and asset returns: A theoretical framework. Econometrica 57(4), 937-69.

Guvenen, F. (2005). Reconciling conflicting evidence on the elasticity of intertemporal substitution: A macroeconomic perspective. Journal of Monetary Economics, forthcoming. 
Hall, R. (1988). Intertemporal substitution in consumption. Journal of Political Economy. 96, 339-357.

Hamilton, J. (1994). Time Series Analysis. Princeton University Press.

Hansen, L. (1982). Large sample properties of generalized method of moments estimators. Econometrica 50, 1929-1954.

Hansen, L., J. Heaton, and N. Li (2004). Consumption strikes back? Working Paper.

Hansen, L. P. and R. Jagannathan (1991). Implications of security market data for models of dynamic economies. Journal of Political Economy 99, 225-262.

Hansen, L. P. and T. J. Sargent (2006). Fragile beliefs and the price of model uncertainty. $N Y U$, unpublished manuscript.

Kan, R. (1995). Structure of pareto optima when agents have stochastic recursive preferences. Journal of Economic Theory 66(2), 626-31.

Kiku, D. (2006). Is the value premium a puzzle? Duke, unpublished manuscript.

Lewis, K. (1999). Trying to explain home bias in equities and consumption. Journal of Economic Literature 37, 571-608.

Lucas, R. (1978). Asset prices in an exchange economy. Econometrica 46 (6), 1429-1445.

Ludvigson, S., M. Lettau, and J. A. Wachter (2004). The declining equity premium: What role does macroeconomic risk play? NYU mimeo.

Lustig, H. and S. Van Nieuwerburgh (2005). The returns on human capital: good news on wall street is bad news on main street. NYU mimeo.

Lustig, H. and A. Verdelhan (2006a). The cross-section of foreign currency risk premia and us consumption growth risk. forthcoming in the American Economic Review.

Lustig, H. and A. Verdelhan (April-May 2006b). The cross-section of foreign currency risk premia and us consumption growth risk. Journal of the European Economic Association, Papers and Proceedings 4.

Mehra, R. and E. Prescott (1985). The equity premium: A puzzle. Journal of Monetary Economics 15, 145-161.

Obstfeld, M. and K. Rogoff (1996). Foundations of International Macroeconomics. MIT Press.

Piazzesi, M. and M. Schneider (2006). Equilibrium yield curves. forthcoming in NBER Macroeconomics Annual 2006, Volume 21. Daron Acemoglu, Kenneth Rogoff and Michael Woodford, Editors, The MIT Press.

Tallarini, T. (2000). Risk-sensitive real business cycles. Journal of Monetary Economics 45, 507-532. 


\section{Appendix A. Derivation of moments}

We express the relevant moments of the variables of the model as a function of the set $\Upsilon$ of deeper parameters defined as $\Upsilon=\left\{\theta, \psi, \delta, \rho_{x}, \sigma_{c}, \sigma_{x}, \rho_{c}^{h f}, \rho_{x}^{h f}\right\}$. We assume that:

$$
\begin{aligned}
\Delta c_{t+1}^{i} & =x_{t}^{i}+\varepsilon_{c, t+1}^{i}, \quad \varepsilon_{c, t}^{i} \sim_{i i d} \mathcal{N}\left(0, \sigma_{c}^{2}\right) \\
x_{t+1}^{i} & =\rho_{x}^{i} x_{t}^{i}+\varepsilon_{x, t+1}^{i}, \quad \varepsilon_{x, t}^{i} \sim_{i i d} \mathcal{N}\left(0, \sigma_{x}^{2}\right), \quad \forall i \in\{h, f\}
\end{aligned}
$$

The Euler equation for the asset that pays one unit of the consumption bundle at each period is:

$$
1=E_{t}\left[M_{t+1}^{i} R_{c, t+1}^{i}\right]
$$

with $\log M_{t+1}=\theta \log \delta-\frac{\theta}{\psi} \log \left(\frac{C_{t+1}^{i}}{C_{t}^{i}}\right)+(\theta-1) \log R_{c, t+1}^{i}$. The return on the consumption asset can be expressed in terms of the price-consumption ratio $v_{c, t}$ as $R_{c, t+1}^{i}=\frac{v_{c, t+1}^{i}+1}{v_{c, t}^{i}} \exp \Delta c_{t+1}^{i}, \forall i \in\{h, f\}$. This implies that (A.2) can be written as:

$$
\left(v_{c, t}^{i}\right)^{\theta}=E_{t}\left[\delta e^{\Delta c_{t+1}^{i}(1-\gamma)}\left(1+v_{c, t+1}^{i}\right)^{\theta}\right]
$$

Linearizing (A.3) around the steady state of $v_{c, t}$ defined as $v_{c}^{i, s s}=\frac{\delta^{i}}{1-\delta^{i}}$, we obtain:

$$
v_{c, t}^{i}=E_{t}\left[\delta+\frac{\delta}{1-\delta}\left(1-\frac{1}{\psi}\right) \Delta c_{t+1}^{i}+\delta v_{c, t+1}^{i}\right]
$$

that can be solved forward delivering the price-consumption ratio as a function of the state variable $x_{t}^{i}$ nd of the deeper parameters of the model:

$$
v_{c, t}^{i}=\alpha_{c}^{i}+\beta_{c}^{i} x_{t}^{i}
$$

with $\alpha_{c}^{i}=\frac{\delta^{i}}{1-\delta^{i}}$ and $\beta_{c}^{i}=\frac{\frac{\delta^{i}}{1-\delta^{i}}\left(1-\frac{1}{\psi^{i}}\right)}{1-\rho_{x}^{i} \delta^{i}}, \forall i \in\{h, f\}$. Log-returns on the assets that pay a stream of consumption follow immediately from (A.1 ) and (A.4):

$$
r_{c, t+1}^{i}=-\log (\delta)+\left[\frac{1}{\psi}\right] x_{t}^{i}+\left[\delta\left(1-\frac{1}{\psi}\right) \frac{1}{1-\rho_{x} \delta}\right] \epsilon_{x, t+1}^{i}+\epsilon_{c, t+1}^{i}
$$

Also the log-stochastic discount factor is:

$$
m_{t+1}^{i}=\log \delta-\frac{1}{\psi} x_{t}^{i}-\gamma \epsilon_{c, t+1}^{i}+\frac{\delta(1-\gamma \Psi)}{\psi\left(1-\rho_{x} \delta\right)} \epsilon_{x, t+1}^{i}
$$

The log-risk free rate $r_{f}^{i}$ is obtained as the solution of:

$$
\begin{aligned}
r_{f}^{i} & =-E_{t}\left[\delta^{\theta} \exp \left\{-\frac{\theta}{\psi} \Delta c_{t+1}^{i}+(\theta-1) r_{c, t+1}^{i}\right\}\right] \\
& =-\log (\delta)+\frac{1}{2}\left[[\gamma]^{2} \sigma_{c}^{2}+\left[\delta\left(1-\frac{1}{\psi}\right) \frac{1}{1-\rho_{x} \delta}\right]^{2} \sigma_{x}^{2}\right]+\frac{1}{\psi} x_{t}^{i}
\end{aligned}
$$


The following moments follow directly from the previous formulas and from the assumption that both countries share the same parametrization:

$$
\begin{aligned}
\operatorname{Var}(\pi) & =2\left[\left(1-\rho_{x}^{h f}\right) \Gamma_{0} \sigma_{x}^{2}+\left(1-\rho_{c}^{h f}\right) \gamma^{2} \sigma_{c}^{2}\right] \\
\operatorname{corr}\left(m_{t}^{h}, m_{t}^{f}\right) & =\frac{\Gamma_{0} \rho_{x}^{h f} \sigma_{x}^{2}+\gamma^{2} \rho_{c}^{h f} \sigma_{c}^{2}}{\Gamma_{0} \sigma_{x}^{2}+\gamma^{2} \sigma_{c}^{2}} \\
\operatorname{Var}\left(r_{c, t+1}^{i}\right) & =\Gamma_{0} \sigma_{x}^{2}+\sigma_{c}^{2} \\
\operatorname{Cov}\left(r_{c, t+1}^{h}, r_{c, t+1}^{f}\right) & =\Gamma_{0} \rho_{x}^{h f} \sigma_{x}^{2}+\rho_{c}^{h f} \sigma_{c}^{2} \\
\operatorname{Cov}\left(\Delta c_{t+1}^{i}, r_{c, t+1}^{i}\right) & =\Gamma_{1}+\sigma_{c}^{2} \\
\operatorname{Cov}\left(\Delta c_{t+1}^{h}, r_{c, t+1}^{f}\right) & =\Gamma_{1} \rho_{x}^{h f}+\rho_{c}^{h f} \sigma_{c}^{2}
\end{aligned}
$$

where $\Gamma_{0}=\left(\frac{1}{\psi}\right)^{2} \frac{1}{1-\rho_{x}^{2}}+\left[\frac{\delta(1-\gamma \psi)}{\psi\left(1-\rho_{x} \delta\right)}\right]^{2}$ and $\Gamma_{1}=\frac{\sigma_{x}^{2}}{\psi\left(1-\rho_{x}^{2}\right)}$.

\section{Appendix B. Proof of Propositions}

Proof of Proposition ??. Assuming complete markets, the correlation $\rho_{m^{h}, m^{f}}$ is given by equation (3). The problem to solved takes the following form:

$$
\begin{aligned}
\text { choose } & \sigma_{m^{h}}, \sigma_{m^{f}} \\
\text { to } & \min \rho_{m^{h}, m^{f}} \\
\text { s.t. } & \sigma_{m^{h}} \geq \underline{\sigma}_{m^{h}} \\
& \sigma_{m^{f}} \geq \underline{\sigma}_{m^{f}}
\end{aligned}
$$

where $\underline{\sigma}_{m^{h}}$ and $\underline{\sigma}_{m^{f}}$ are the Hansen and Jagannathan (1991) bounds. Attaching Lagrange multipliers $\lambda^{h}$ and $\lambda^{f}$ to the two constraints, the first order necessary conditions are:

$$
\begin{aligned}
& \frac{\partial \rho_{m^{h}, m^{f}}}{\partial \sigma_{m^{h}}}-\lambda^{h}=0 \\
& \frac{\partial \rho_{m^{h}, m^{f}}}{\partial \sigma_{m^{f}}}-\lambda^{f}=0
\end{aligned}
$$

along with the two constraints. Four cases must be taken into account.

Case 1: $\lambda^{h}=\lambda^{f}=0$. This implies $\sigma_{\pi}^{2}=0$, a contradiction.

Case 2: $\lambda^{h}=0, \lambda^{f}>0$. The system of first order conditions implies:

$$
\left\{\begin{array}{l}
\sigma_{m f}=\underline{\sigma}_{m f} \\
\sigma_{m^{h}}^{2}=\underline{\sigma}_{m f}^{2}-\sigma_{\pi}^{2} \geq \underline{\sigma}_{m^{h}}^{2} \quad \Rightarrow \underline{\sigma}_{m^{f}}^{2} \geq \sigma_{\pi}^{2}+\underline{\sigma}_{m^{h}}^{2} \\
\frac{\underline{\sigma}_{m f}^{2}-\left(\underline{\sigma}_{m f}^{2}-\sigma_{\pi}^{2}\right)+\sigma_{\pi}^{2}}{2 \underline{\sigma}_{m f}^{2} \sqrt{{\underline{\sigma_{m}^{2}}}_{m}^{2}-\sigma_{\pi}^{2}}}-\lambda^{f}=0 \Rightarrow \lambda^{f}>0
\end{array}\right.
$$

that is, if $\underline{\sigma}_{m^{f}}^{2} \geq \sigma_{\pi}^{2}+\underline{\sigma}_{m^{h}}^{2}$, the minimum is achieved for $\sigma_{m^{f}}=\underline{\sigma}_{m^{f}}$ and $\sigma_{m^{h}}=\sqrt{\underline{\sigma}_{m^{f}}^{2}-\sigma_{\pi}^{2}}$.

Case 3: $\lambda^{h}>0, \lambda^{f}=0$. This case is symmetric to the previous one. 
Case 4: $\lambda^{h}>0, \lambda^{f}>0$. The system of first order conditions implies:

$$
\left\{\begin{array}{l}
\sigma_{m} f=\underline{\sigma}_{m} \\
\sigma_{m^{h}}=\underline{\sigma}_{m^{h}} \\
\frac{\underline{\sigma}_{m f}^{2}-\underline{\sigma}_{m}^{2}+\sigma_{\pi}^{2}}{2 \underline{\sigma}_{m f}^{2} \underline{\sigma}_{m}^{h}}-\lambda^{f}=0 \\
\frac{\underline{\sigma}_{m}^{2}-\underline{\sigma}_{m f}^{2}+\sigma_{\pi}^{2}}{2 \underline{\sigma}_{m}^{2} \underline{\sigma}_{m} f}-\lambda^{h}=0
\end{array}\right.
$$

that is, if $\underline{\sigma}_{m^{f}} \in\left(\underline{\sigma}_{m^{h}}-\sigma_{\pi}^{2}, \underline{\sigma}_{m^{h}}+\sigma_{\pi}^{2}\right)$ the minimizer is the Hansen and Jagannathan (1991) bound itself.

Combining the four cases, we obtain:

$$
\underline{\rho}_{m^{h}, m^{f}}=\left\{\begin{array}{lll}
\frac{\underline{\sigma}_{m^{h}}^{2}-\sigma_{\pi}^{2}}{\underline{\sigma}_{m^{h}} \sqrt{\underline{\sigma}_{m^{h}}-\sigma_{\pi}^{2}}} & \text { if } & \underline{\sigma}_{m^{f}} \leq \underline{\sigma}_{m^{h}}-\sigma_{\pi}^{2} \\
\frac{\underline{\sigma}_{m h}^{2}+\underline{\sigma}_{m f}^{2}-\sigma_{\pi}^{2}}{2 \underline{\sigma}_{m}^{h} \underline{\sigma}_{m} f} & \text { if } & \underline{\sigma}_{m^{f}} \in\left(\underline{\sigma}_{m^{h}}-\sigma_{\pi}^{2}, \underline{\sigma}_{m^{h}}+\sigma_{\pi}^{2}\right) \\
\frac{\underline{\sigma}_{m f}^{2}-\sigma_{\pi}^{2}}{\underline{\sigma}_{m} \sqrt{\underline{\sigma}_{m}-\sigma_{\pi}^{2}}} & \text { if } & \underline{\sigma}_{m^{f}} \geq \underline{\sigma}_{m^{h}}-\sigma_{\pi}^{2}
\end{array}\right.
$$

That concludes the proof.

Proof of Proposition 2. For any choice of the parameters that satisfy $\rho_{x}^{i} \neq 1$ and $\rho_{x}^{i} \delta^{i} \neq 1$ the following three partial derivatives

$$
\begin{aligned}
\frac{\partial \operatorname{corr}\left(m_{t}^{h}, m_{t}^{f}\right)}{\partial \rho_{x}^{h f}} & =\frac{\Gamma_{0} \sigma_{x}^{2}}{\Gamma_{0} \sigma_{x}^{2}+\gamma^{2} \sigma_{c}^{2}}>0 \\
\frac{\partial \operatorname{corr}\left(m_{t}^{h}, m_{t}^{f}\right)}{\partial \Psi} & =-\frac{2 \Gamma_{0} \gamma^{2}\left(\rho_{x}^{h f}-\rho_{c}^{h f}\right) \sigma_{x}^{2} \sigma_{c}^{2}}{\psi\left(\Gamma_{0} \sigma_{x}^{2}+\gamma^{2} \sigma_{c}^{2}\right)^{2}} \\
\frac{\partial \operatorname{corr}\left(m_{t}^{h}, m_{t}^{f}\right)}{\partial \rho_{x}} & =\frac{2\left[\frac{\rho_{x}}{\left(1-\rho_{x}^{2}\right)^{2}}+\frac{\delta^{3}(1-\gamma \psi)^{2}}{\left(1-\rho_{x} \delta\right)^{3}}\right] \gamma^{2}\left(\rho_{x}^{h f}-\rho_{c}^{h f}\right) \sigma_{x}^{2} \sigma_{c}^{2}}{\psi^{2}\left[\Gamma_{0} \sigma_{x}^{2}+\gamma^{2} \sigma_{c}^{2}\right]^{2}}
\end{aligned}
$$

exist and are well defined. (B.1) is positive for all the values of the parameters that respect the two conditions, implying that the correlation of the two stochastic discount factors is strictly increasing with respect to $\rho_{x}^{h f}$. When $\rho_{x}^{h f}=\rho_{c}^{h f}$ (B.2) is always zero, meaning that changes in $\Psi, \gamma$ or $\delta$ do not affect the correlation of the two stochastic discount factors. If $\rho_{x}^{h f} \neq \rho_{c}^{h f}$, this derivative is zero only if $\psi=\frac{1}{\gamma} \widetilde{\delta}$, where $\widetilde{\delta}=\frac{1-2 \rho_{x} \delta+\delta^{2}}{\delta^{2}\left(1-\rho_{x}^{2}\right)}$. In particular, when $\rho_{x}^{h f}>\rho_{c}^{h f}$ the sign of the derivative is positive for $\Psi>\frac{1}{\gamma} \widetilde{\delta}$ and negative for $\psi<\frac{1}{\gamma} \widetilde{\delta}$. Notice that

$$
\lim _{\delta \rightarrow 1^{-}} \frac{1}{\gamma} \widetilde{\delta} \geq \frac{1}{\gamma} \quad \text { and } \quad \lim _{\rho_{x} \rightarrow 1^{-} \delta \rightarrow 1^{-}} \lim _{\gamma} \frac{1}{\gamma} \widetilde{\delta}=\frac{1}{\gamma}
$$

So, for a high persistence of the long run component and an individual discount factor close to one, the minimum of the cross correlation of the two discount factors is achieved for $\psi=\frac{1}{\gamma}$, that is when the Epstein-Zin preferences collapse to the standard CES utility function. (B.3) is always 
positive when $\rho_{x}^{h f}>\rho_{c}^{h f}$, negative when $\rho_{x}^{h f}<\rho_{c}^{h f}$ and equal to zero when $\rho_{x}^{h f}=\rho_{c}^{h f}$. Therefore if $\rho_{x}^{h f}>\rho_{c}^{h f}$, the minimum is achieved for $\rho_{x}=0$. When $\rho_{x}^{h f}>\rho_{c}^{h f}$, (B.1), (B.2) and (B.3) imply the existence of one unique minimizer at $\left(\rho_{x}=0, \rho_{x}^{h f}=0, \psi=\frac{1}{\gamma} \widetilde{\delta}\right)$.

Proof of Proposition 3. The partial derivative of (A.8) with respect to $\rho_{x}^{h f}$ exists and is well defined provided that $\rho_{x} \neq 1$ and $\rho_{x} \delta \neq 1$ :

$$
\frac{\partial \operatorname{Var}(\pi)}{\partial \rho_{x}^{h f}}=-2\left[\left(\frac{1}{\psi}\right)^{2} \frac{1}{1-\rho_{x}^{2}}+\left[\frac{\delta(1-\gamma \psi)}{\psi\left(1-\rho_{x} \delta\right)}\right]^{2}\right] \sigma_{x}^{2} \leq 0
$$

In particular, this derivative is always negative, implying that the volatility of the log-depreciation rate achieves its minimum when $\rho_{x}^{h f}=1$.

\section{Appendix C. Numerical algorithm for the approximation of the price dividend ratios}

We describe the procedure to numerically approximate the price-consumption and price-dividend ratios for the most general case in which stochastic volatility is in the model, too. We discretize the support of $x$ and $\sigma$ into $I_{x}$ and $I_{\sigma}$ points respectively, to get $I=I_{x} \cdot I_{\sigma}$ nodes $(x, \sigma)_{i}, \forall i=1, \ldots, I$. In what follows, we will refer to $x_{i}$ and $\sigma_{i}$ as the first and the second entry of $(\alpha, s)_{i}$ respectively. Specify $J$ known linearly independent basis functions $\phi_{j}\left((x, \sigma)_{i}\right), j \in\{1, \ldots, J\}$. In our solution, we employ a third order polynomial in $x$ combined with a first order polynomial in $\sigma$, implying that $J=6$. The goal is to find basis coefficients $c_{j}, j=1, \ldots, J$ that best approximate the Euler equation

$$
v_{c}^{i}=V\left((x, \sigma)_{i}\right) \approx \sum_{j=1}^{J} c_{j} \phi_{j}\left((x, \sigma)_{i}\right)=\sum_{j=1}^{J} c_{j} \phi_{j, i}
$$

$\forall i=1, \ldots, I$ or, in the equivalent matrix notation:

$$
v_{c} \approx \Phi c
$$

where $v_{c}$ is the $I \times 1$ vector of approximated value functions at each node, $\Phi$ is the $I \times J$ collocation matrix and $c=\left[c_{1}, \ldots, c_{J}\right]^{\prime}$ is the vector of approximation coefficients. We also discretize the support of the three shocks in $K_{1}, K_{2}$ and $K_{3}$ points and denote $w_{1, k}, w_{2, k}$ and $w_{3, k}$ the approximated probability masses associated to each of the nodes. The shocks are assumed to independent. Under 
these assumptions, we get for each node $i \in\{1, \ldots, I\}$ :

$$
\begin{aligned}
v_{c, i}= & \delta\left[\sum_{\varepsilon_{c} \in K_{1}} \sum_{\varepsilon_{x} \in K_{2}} \sum_{\varepsilon_{\sigma} \in K_{3}} w_{1, \varepsilon_{c}} w_{2, \varepsilon_{x}} w_{3, \varepsilon_{\sigma}} \exp \left\{\theta\left(1-\frac{1}{\psi}\right) \Delta c_{i, \varepsilon_{c}}^{\prime}\right\}\right. \\
& \left.\left(1+\sum_{j=1}^{J} c_{j} \phi_{j}\left(x_{i, \varepsilon_{x}}^{\prime}, \sigma_{i, \varepsilon_{\sigma}}^{\prime}\right)\right)^{\theta}\right]^{\frac{1}{\theta}}
\end{aligned}
$$

where

$$
\begin{aligned}
\sigma_{i, \varepsilon_{\sigma}}^{\prime} & =\bar{\sigma}+\nu_{1}\left(\sigma_{i}-\bar{\sigma}\right)+\sigma_{w} \varepsilon_{\sigma} \\
\Delta c_{i, \varepsilon_{c}}^{\prime} & =x_{i}+\sigma_{i, \varepsilon_{\sigma}}^{\prime} \varepsilon_{c} \\
x_{i, \varepsilon_{x}}^{\prime} & =\rho_{x} x_{i}+\varphi_{e} \sigma_{i, \varepsilon_{\sigma}}^{\prime} \varepsilon_{x}
\end{aligned}
$$

We can now use the following algorithm to solve the Euler equation recursively:

1. guess an initial vector of basis coefficients $c^{1}$

2. for each node $(s, \alpha)_{i}$ compute the right hand side of equation (C.2) using $c^{1}$ and call $v\left(c^{1}\right)$ the outcome

3. solve for $c^{2}=\left(\Phi^{\prime} \Phi\right)^{-1} \Phi^{\prime} v\left(c^{1}\right)$

4. replace $c^{1}$ with $c^{2}$ and iterate until convergence.

Having solved for the price-consumption ratio $v_{c}$, we can solve the Euler equation for the pricedividend ratio in a similar way:

$$
\begin{aligned}
v_{d, i}= & \delta^{\theta} \sum_{\varepsilon_{c} \in K_{1}} \sum_{\varepsilon_{x} \in K_{2}} \sum_{\varepsilon_{\sigma} \in K_{3}} \sum_{\varepsilon_{d} \in K_{1}} w_{1, \varepsilon_{c}} w_{2, \varepsilon_{x}} w_{3, \varepsilon_{\sigma}} w_{4, \varepsilon_{d}} \exp \left\{m_{i,\left(\varepsilon_{c}, \varepsilon_{x}, \varepsilon_{\sigma}\right)}^{\prime}\right\} \\
& \left(1+\sum_{j=1}^{J} d_{j} \phi_{j}\left(x_{i, \varepsilon_{x}}^{\prime}, \sigma_{i, \varepsilon_{\sigma}}^{\prime}\right)\right)
\end{aligned}
$$

where

$$
m_{i,\left(\varepsilon_{c}, \varepsilon_{x}, \varepsilon_{\sigma}\right)}^{\prime}=\left(\theta-1-\frac{\theta}{\psi}\right) \Delta c_{i, \varepsilon_{c}}^{\prime}(\theta-1) \log \left(\frac{1+v_{i,\left(\varepsilon_{c}, \varepsilon_{x}, \varepsilon_{\sigma}\right)}^{\prime}}{v_{i}}\right)
$$

\section{Appendix D. Spectral analysis}

Denoting as $Y_{t}=\left[\begin{array}{cc}\Delta c_{t}^{h} & \Delta c_{t}^{f}\end{array}\right]$ the vector of consumption growth in the US and the UK at time $t$, the population spectrum at frequency $\omega$ is

$$
S_{Y}(\omega)=\frac{1}{2 \pi} \sum_{k=-\infty}^{k=+\infty} \Gamma_{k} e^{-i k \omega}
$$


where $\Gamma_{j}=E\left[Y_{t}-\bar{Y}\right]\left[Y_{t-j}-\bar{Y}\right]^{\prime}$ and $\bar{Y}=E\left[Y_{t}\right]$. We follow Hamilton (1994) in estimating $\Gamma_{j}, \forall j$ with their sample counterparts:

$$
\widehat{\Gamma}_{j}=\frac{\sum_{t=j+1}^{T}\left(Y_{t}-\bar{Y}\right)\left(Y_{t}-\bar{Y}\right)^{\prime}}{T}
$$

and smoothing (D.1) with a 10 period Bartlett (1964) window

$$
\widehat{S}_{Y}(\omega)=\frac{1}{2 \pi} \sum_{k=-10}^{k=10}\left(1-\frac{|k|}{10}\right) \widehat{\Gamma}_{k} e^{-i \omega k}
$$

As a measure of the covariance explained at different frequencies, we consider the coherence, defined as:

$$
K_{\Delta c^{h}, \Delta c^{f}}^{2}(\omega)=\frac{\left|\widehat{S}_{\Delta c^{h}, \Delta c^{f}}(\omega)\right|^{2}}{\widehat{S}_{\Delta c^{h}}(\omega) \widehat{S}_{\Delta c^{f}}(\omega)}
$$

\section{Appendix E. Details of the estimation}

The GMM is based on the following 46 unconditional moments:

- $\operatorname{cov}\left(\Delta c_{t}^{i}, \Delta c_{t-j}^{i}\right), \forall i \in\{h, f\} \wedge j \in\{0,1, \ldots, 4\}$

- $\operatorname{cov}\left(\Delta c_{t}^{h}, \Delta c_{t-j}^{f}\right), \forall j \in\{0,1, \ldots, 4\}$

- $\operatorname{cov}\left(\Delta c_{t}^{f}, \Delta c_{t-j}^{h}\right), \forall j \in\{1, \ldots, 4\}$

- $\operatorname{cov}\left(\Delta d_{t}^{i}, \Delta d_{t-j}^{i}\right), \forall i \in\{h, f\} \wedge j \in\{0,1, \ldots, 4\}$

- $\operatorname{cov}\left(\Delta d_{t}^{h}, \Delta d_{t-j}^{f}\right), \forall j \in\{0,1,2\}$

- $\operatorname{cov}\left(\Delta d_{t}^{f}, \Delta d_{t-j}^{h}\right), \forall j \in\{0,1,2\}$

- $\operatorname{Var}\left(\frac{e_{t+1}}{e_{t}}\right)$

- $E\left[r_{f, t}^{i}\right], \operatorname{Var}\left[r_{f, t}^{i}\right]$ and $\operatorname{cov}\left[r_{f, t}^{i}, r_{f, t-1}^{i}\right], \forall i \in\{h, f\}$

- $E\left[\left(r_{d, t}^{i}-r_{f, t}^{i}\right)\right]$ and $\operatorname{Var}\left[\left(r_{d, t}^{i}-r_{f, t}^{i}\right)\right], \forall i \in\{h, f\}$

- $\operatorname{cov}\left[\left(r_{d, t}^{h}-r_{f, t}^{h}\right),\left(r_{d, t}^{f}-r_{f, t}^{f}\right)\right]$

To better account for the non-linearities in the dynamics of asset returns, we approximate the model around a stochastic steady state. The formulas derived in this appendix are then used to 
construct the relevant moments that are used in the GMM estimation.

Let

$$
\left[\begin{array}{lll}
\varepsilon_{c, t} & \varepsilon_{d, t} & \varepsilon_{x, t}
\end{array}\right] \sim N(0, S)
$$

The Campbell and Shiller (1988) log linearization of the returns implies:

$$
\begin{aligned}
v_{c, t} & =\bar{v}_{c}+\sum_{i=0}^{\infty} \kappa_{c} E_{t}\left[\Delta c_{t+1+i}\right]-\sum_{i=0}^{\infty} \kappa_{c}^{i} E_{t}\left[r_{c, t+1+i}\right] \\
\kappa_{c} & \equiv \frac{\overline{\exp \left(v_{c}\right)}}{1+\overline{\exp \left(v_{c}\right)}}
\end{aligned}
$$

When Epstein and Zin (1989) preferences are adopted, a log-linearization of the first order conditions of the representative agent implies:

$$
\begin{aligned}
m_{t+1} & =\bar{m}-\frac{1}{\psi} x_{c, t}-\kappa_{c} \frac{\gamma-1 / \psi}{1-\rho \kappa_{c}} \epsilon_{x, t+1}-\gamma \epsilon_{c, t+1} \\
r_{c, t+1} & =\overline{r_{c}}+\frac{1}{\psi} x_{t}+\kappa_{c} \frac{1-1 / \psi}{1-\rho \kappa_{c}} \epsilon_{x, t+1}+\epsilon_{c, t+1} \\
r_{f, t} & =\bar{r}_{f}+\frac{1}{\psi} x_{t} \\
v_{c, t} & =\bar{v}_{c}+\frac{1-1 / \psi}{1-\kappa_{c} \rho} x_{t}
\end{aligned}
$$

Given the results above, the Euler Equation for the asset that pays consumption (evaluated at $\left.x_{t}=0\right)$ provides the following non linear equation in $\kappa_{c}$ :

$$
\kappa_{c}=\delta \exp \left\{\left(1-\frac{1}{\psi}\right)\left(\mu-.5(\gamma-1) \operatorname{Var}\left[\epsilon_{c, t+1}+\kappa_{c}\left(1-\rho \kappa_{c}\right)^{-1} \epsilon_{x, t+1}\right]\right)\right\}
$$

Rewriting the stochastic discount factor $m_{t+1}$ and the return in vector form, we obtain:

$$
\begin{aligned}
m_{t+1} & =\bar{m}-\frac{1}{\Psi} x_{c, t}+\Gamma_{m} \epsilon_{t+1} \\
\Gamma_{m} & \equiv\left[\begin{array}{ccc}
-\gamma & 0 & -\kappa_{c} \frac{\gamma-1 / \Psi}{1-\rho \kappa_{c}}
\end{array}\right] \\
r_{c, t+1} & =\overline{r_{c}}+\frac{1}{\Psi} x_{t}+\Gamma_{c} \epsilon_{t+1} \\
\Gamma_{c} & \equiv\left[\begin{array}{lll}
1 & 0 & \kappa_{c} \frac{1-1 / \Psi}{1-\rho \kappa_{c}}
\end{array}\right]
\end{aligned}
$$

Since

$$
E_{t}\left[r_{c, t+1}^{e x}\right]=-\operatorname{cov}\left(m_{t+1}-E_{t}\left[m_{t+1}\right], r_{c, t+1}-E_{t}\left[r_{c, t+1}\right]\right)-.5 V\left(r_{c, t+1}-E_{t}\left[r_{c, t+1}\right]\right)
$$

then the following holds:

$$
E_{t}\left[r_{c, t+1}^{e x}\right]=-\Gamma_{m} C \Gamma_{c}^{\prime}-.5 \Gamma_{c} C \Gamma_{c}^{\prime}
$$


By rearranging the definition of the stochastic discount factor, the following holds:

$$
\begin{aligned}
E\left[r_{f}\right] & =-\log (\delta)+\frac{1}{\Psi} E(\Delta c)+\frac{(1-\theta)}{\theta} E_{t}\left[r_{c, t+1}^{e x}\right]-\frac{1}{2 \theta} V_{t}\left[m_{t+1}\right] \\
& =-\log (\delta)+\frac{1}{\Psi} \mu+\frac{(1-\theta)}{\theta}\left(-\Gamma_{m} C \Gamma_{c}^{\prime}-.5 \Gamma_{c} C \Gamma_{c}^{\prime}\right)-\frac{1}{2 \theta} \Gamma_{m} C \Gamma_{m}^{\prime}
\end{aligned}
$$

We are now able to find the intercept of the log stochastic discount factor:

$$
\bar{m}=\theta \log \delta-\frac{\theta}{\Psi} \mu+(\theta-1)\left(E\left[r_{c}^{e x}\right]+E\left[r_{f}\right]\right)
$$

We follow the same strategy in order to find the log-return of the asset that entitles to the stream of dividends:

$$
\begin{aligned}
v_{d, t} & =\bar{v}_{d}+\frac{\lambda-1 / \psi}{1-\kappa_{d} \rho} x_{t} \\
r_{d, t+1} & =\bar{r}_{d}+\frac{1}{\psi} x_{t}+\kappa_{d} \frac{\lambda-1 / \psi}{1-\rho \kappa_{d}} \epsilon_{x, t+1}+\epsilon_{d, t+1}
\end{aligned}
$$

An equivalent vector form representation is:

$$
\begin{aligned}
r_{d, t+1} & =\bar{r}_{d}+\frac{1}{\psi} x_{t}+\Gamma_{d} \epsilon_{t+1} \\
\Gamma_{d} & \equiv\left[\begin{array}{lll}
0 & 1 & \kappa_{d} \frac{\lambda-1 / \psi}{1-\rho \kappa_{d}}
\end{array}\right]
\end{aligned}
$$

Given the results above, the Euler Equation for the asset that pays dividend (evaluated at $x_{t}=0$ ) provides the following non linear equation in $\kappa_{d}$ :

$$
\kappa_{d}=\exp \left\{\bar{m}+\mu+.5 \operatorname{Var}\left[\left(\Gamma_{m}+\Gamma_{d}\right) v_{t+1}\right]\right\}
$$

The equity premium in this case is:

$$
E_{t}\left[r_{d, t+1}^{e x}\right]=-\Gamma_{m} C \Gamma_{d}^{\prime}-.5 \Gamma_{d} C \Gamma_{d}^{\prime}
$$

All other moments can be easily computed from these formulas. 